\title{
UHRF1-repressed 5'-hydroxymethylcytosine is essential for the male meiotic prophase I
}

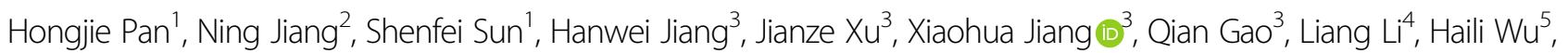
Huajun Zheng', Qi Qi ${ }^{1}$, Tianqi Li ${ }^{1}$, Meixing Zhang ${ }^{1}$, Lingling Zhang ${ }^{1}$, Xiaofeng Wan' ${ }^{1}$, Xinhua Lin², Jiemin Wong ${ }^{4}$, Qinghua Shi ${ }^{3}$ and Runsheng $\mathrm{Li}^{1}$

\begin{abstract}
5'-hydroxymethylcytosine (5hmC), an important 5'-cytosine modification, is altered highly in order in male meiotic prophase. However, the regulatory mechanism of this dynamic change and the function of $5 \mathrm{hmC}$ in meiosis remain largely unknown. Using a knockout mouse model, we showed that UHRF1 regulated male meiosis. UHRF1 deficiency led to failure of meiosis and male infertility. Mechanistically, the deficiency of UHRF1 altered significantly the meiotic gene profile of spermatocytes. Uhrf 1 knockout induced an increase of the global $5 \mathrm{hmC}$ level. The enrichment of hyper-5hmC at transcriptional start sites (TSSs) was highly associated with gene downregulation. In addition, the elevated level of the TET1 enzyme might have contributed to the higher $5 \mathrm{hmC}$ level in the Uhrf1 knockout spermatocytes. Finally, we reported Uhrf1, a key gene in male meiosis, repressed hyper-5hmC by downregulating TET1. Furthermore, UHRF1 facilitated RNA polymerase II (RNA-pol2) loading to promote gene transcription. Thus our study demonstrated a potential regulatory mechanism of $5 \mathrm{hmC}$ dynamic change and its involvement in epigenetic regulation in male meiosis.
\end{abstract}

\section{Introduction}

Meiosis, characterized with a single round of DNA replication followed by two successive divisions, fosters genetic diversity and allows the total DNA content to be maintained through successive generations. In male, meiosis is an essential process of spermatogenesis. Meiotic disorder is one of the major causes of male infertility ${ }^{1,2}$. The progression of meiosis is highly guided by epigenetic transitions ${ }^{3}$.

DNA modification, one of the important epigenetic mechanisms that influence spermatogenesis and male

Correspondence: Qinghua Shi (qshi@ustc.edu.cn) or

Runsheng Li (runshengli2007@163.com)

${ }^{1}$ National Health Commission (NHC) Key Laboratory of Reproduction

Regulation (Shanghai Institute of Planned Parenthood Research), Fudan

University, 200032 Shanghai, P.R. China

${ }^{2}$ State Key Laboratory of Genetic Engineering, Institute of Biostatistics and

Computational Biology, School of Life Sciences, Fudan University, 200438

Shanghai, P.R. China

Full list of author information is available at the end of the article

These authors contributed equally: Hongjie Pan, Ning Jiang

Edited by D. Heery fertility, associates with meiosis functionally ${ }^{4-6}$. DNA methylation, typically in the context of $\mathrm{CPG}$ in the promoter regions and constitutive heterochromatin, is generally associated with reduced gene transcription and silencing ${ }^{7-10}$. Methylation of cytosine at the fifth carbon can be converted into 5'-hydroxymethylcytosine $(5 \mathrm{hmC})$, through consecutive oxidation reactions catalyzed by teneleven-translocation 1, 2, and 3 (TET1, 2, and 3) enzymes. Global detections of $5 \mathrm{hmC}$ in human, mouse, zebrafish, and Xenopus ${ }^{11-19}$ suggest that the dynamics and abundance of $5 \mathrm{hmC}$ are cell-type dependent and developmentally regulated. In mice, germ cells are first specified as primordial germ cells (PGCs) in the developing embryo around embryonic day $6.25(\mathrm{E} 6.25)^{20}$. The first global DNA demethylation takes place in PGCs to reset the epigenome for totipotency. Epigenetic reprogramming (including 5-methylcytosine $(5 \mathrm{mC})$ and $5 \mathrm{hmC}$ ) enables the transition from PGC to gonocyte ${ }^{21}$. In addition, $5 \mathrm{hmC}$ takes roles as a cis element promoting or repressing gene expression, because it can be localized at

\section{(c) The Author(s) 2020}

Open Access This article is licensed under a Creative Commons Attribution 4.0 International License, which permits use, sharing, adaptation, distribution and reproduction in any medium or format, as long as you give appropriate credit to the original author(s) and the source, provide a link to the Creative Commons license, and indicate if changes were made. The images or other third party material in this article are included in the article's Creative Commons license, unless indicated otherwise in a credit line to the material. If material is not included in the article's Creative Commons license and your intended use is not permitted by statutory regulation or exceeds the permitted use, you will need to obtain permission directly from the copyright holder. To view a copy of this license, visit http://creativecommons.org/licenses/by/4.0/. 
enhancer, promoter, transcriptional start site (TSS), gene body, 3' untranslated region (UTR), or intragenic regions. Although the $5 \mathrm{hmC}$ alters in a highly order in germ line during spermatogenesis ${ }^{22}$, to date, the underlying molecular mechanism and physiological function of $5 \mathrm{hmC}$ in germ cells after the PGC period remain not well understood.

A previous study showed that the $5 \mathrm{mC}$ in differentiating spermatogonia was higher than that in undifferentiating spermatogonia. This suggested that spermatogonia underwent a $\mathrm{KIT}^{\text {neg }}$ to $\mathrm{KIT}^{\text {pos }}$ transition accompanied with an enhancement of DNA methylation. They also pointed out that the DNA methylation might regulate directly or indirectly the genes guiding spermatogonial differentiation (for example, Plzf and Kit) ${ }^{23}$. One of the key player of DNA methylation is UHRF1, a multifunctional protein ${ }^{24-26}$, which is essential for maintenance or de novo DNA methylation ${ }^{27-34}$. However, the role of UHRF1 in male germ cells after the spermatogonia differentiation, for example, meiosis, remains largely unknown. A structural analysis showed UHRF1 interacted directly and to a similar degree with $5 \mathrm{mC}$ or $5 \mathrm{hmC}^{35}$. Although it has been well established that UHRF1 mediates DNA methylation, its function linking to $5 \mathrm{hmC}$ is still in its infancy.

Here we showed that UHRF1 controlled the meiosis of male mice. Knockout of Uhrf1 in mice resulted in a serious of impairment of meiotic events, like deficient synaptonemal complex (SC) assembly, skewed meiotic recombination, and failed $\mathrm{H} 1 \mathrm{t}$ incorporation. In this study, we identified a previously unknown role that UHRF1 repressed $5 \mathrm{hmC}$ and facilitated the RNA-pol2 binding to DNA. Benefiting from conditional knockout mice, we provided a potential regulatory mechanism for the $5 \mathrm{hmC}$ dynamic change and demonstrated the physiological function of $5 \mathrm{hmC}$ in meiosis.

\section{Materials and methods}

Mice

The C57BL/6 Uhrf1-flox mouse strain was prepared as mentioned by the method of gene trap. The C57BL/6 Stra8-cre mouse strain, which expressed knock-in CRE recombinase driven by endogenous Stra 8 promoter ${ }^{36}$, was provided as a gift by Professor Minghan Tong at the Institute of Biochemistry and Cell Biology, CAS. All mice were kept under the controlled photoperiod conditions (lights on 07:00-19:00 hours) and supplied with food and sterilized $\mathrm{H}_{2} \mathrm{O}$ ad libitum. All experiments were conformed to the regulations drafted by Association for Assessment and Accreditation of Laboratory Animal Care in Shanghai and were approved by the Shanghai Institute of Planned Parenthood Research Center for Animal Research. To generate the Uhrff ${ }^{f / f}$;Stra8-cre male mice, we intercrossed female Uhrf1 $1^{f / f}$ and male $U h r f 1^{f /+}$;Stra8- cre mice. Genomic DNA was extracted from tail biopsies and analyzed using the TaKaRa Taq ${ }^{\mathrm{TM}}$ Hot version (Cat\# R007A). Primers for genotyping were listed in Table S1.

\section{Cell, plasmids, and transfection}

GC1 cells (ATCC, Cat\# CRL-2053) were cultured in high-glucose Dulbecco's Modified Eagle's Medium (DMEM) (GIBCO, 11965-092) with 10\% fetal bovine serum (GIBCO, 10437028), $100 \mathrm{U} / \mathrm{mL}$ penicillin, and $100 \mathrm{mg} / \mathrm{mL}$ streptomycin (GIBCO, 15140122) and maintained at $5 \% \mathrm{CO}_{2}$. Plasmids (pcDNA3.1-UHRF1-Flag, pcDNA3.1- ${ }_{m} U H R F 1-\triangle S R A-F l a g, \quad$ pcDNA3.1 ${ }_{m} U H R F 1-$ $\triangle T T D$-Flag, and pcDNA3.1- ${ }_{m}$ UHRF1-ARING-Flag) were from Jiemin Wong's laboratory. Cells were passaged 2-3 times after thawing and transfected at $70-80 \%$ confluency. Transfection of plasmids was performed using lipofectamine 3000 (Invitrogen, L3000001). Detection of mycoplasma in $\mathrm{GC}-1$ cell line using polymerase chain reaction (PCR) methods was performed as previously described ${ }^{37}$.

\section{Hematoxylin and eosin (H\&E) staining and immunohistochemistry (IHC)}

Testes were collected and fixed immediately in Bouin's solution for $\mathrm{H} \& \mathrm{E}$ staining and in $4 \%$ paraformaldehyde (PFA) for IHC. For IHC assay, sections $(4-5 \mu \mathrm{m})$ were deparaffinized in xylene and rehydrated in gradient alcohols. After antigen retrieval, the sections were denatured or not with $2 \mathrm{~N} \mathrm{HCl}$, at $37^{\circ} \mathrm{C}$, for $30 \mathrm{~min}$, followed by blocking. Sections were then incubated with primary antibodies overnight at $4{ }^{\circ} \mathrm{C}$. The sections were incubated with secondary antibodies for $20 \mathrm{~min}$ the next day and then developed with 3,3'-diaminobenzidine and counterstained with hematoxylin. Antibodies were diluted as follows: 5-mC at 1:100 (Active Motif, Cat\# 39649), 5-hmC at 1:100 (Active Motif Cat\# 39769), TET1 at 1:100 (Millipore Cat\# 09-872), TET2 at 1:100 (Millipore Cat\# ABE364), TET3 at 1:100 (Millipore Cat\# MABE1133), and UHRF1 at 1:200 (Active Motif, Cat\# 61341). To ensure reproducibility of the results, samples from $\geq 3$ animals were evaluated.

\section{Meiotic prophase cell spreading and immunofluorescence staining}

Spreads of spermatocytes and immunofluorescence staining were prepared according to the previous references $^{38,39}$. Briefly, seminiferous tubules were incubated in hypotonic extraction buffer $(50 \mathrm{mM}$ Sucrose, $17 \mathrm{mM}$ Sodium citrate, $30 \mathrm{mM}$ Tris ( $\mathrm{pH} 8.2$ ), $2.5 \mathrm{mM}$ dithiothreitol, $1 \mathrm{mM}$ phenylmethylsulfonyl fluoride $(\mathrm{pH} 8.3)$, and $5 \mathrm{mM}$ EDTA) on ice for $20 \mathrm{~min}$, minced in $100 \mathrm{mM}$ sucrose, spread on slides, and fixed in $1 \%$ PFA with $0.1 \%$ Triton X-100. Slides were incubated in a humid chamber overnight, dried, and washed in phosphate-buffered saline (PBS) and water containing Photoflo (Kodak, NY, USA). 
Following blocking in 10\% donkey serum and 3\% bovine serum albumin, immunofluorescence staining was performed by incubating with the primary antibodies: $\gamma \mathrm{H} 2 \mathrm{AX}$ (1:1000; Novus, Cat\# NB100-384), SYCP3 (1:100; Abcam, Cat\# ab97672 or Novus, Cat\# NB300-232), DMC1 (1:100, Santa Cruz, Cat\# sc-22768), MLH1 (1:100, BD, Cat\# 551092), SYCP1 (1:100, NOVAS, Cat\# NB300-229), and RNA-Polymerase II (1:200, Active Motif, Cat\# 39097), overnight at room temperature. Alexa 488 (1:400, Thermo Fisher) or Alexa 555 (1:200, Thermo Fisher) fluorescent secondary antibody was used. Slides were incubated with secondary antibodies at $37^{\circ} \mathrm{C}$ for $1 \mathrm{~h}$ in dark, washed, and mounted with Vecta shield cover slips (Vector Laboratories, Cat\# H-1000).

\section{Primary germ cell preparation}

Testicular cells were obtained as previously described ${ }^{40}$. Briefly, the capsules of the testis were removed and testicular tubules were minced and transferred to a $50-\mathrm{mL}$ Falcon tube. Tissue was suspended in F12/DMEM (Gibco), centrifuged, collected, and then subjected to digestion. Trypsin/EDTA $(0.1 \mathrm{mg} / \mathrm{mL}$; Sigma $)$, DNase (0.02 mg/mL; Sigma), glycine (1 M; Sigma), EDTA (2 mM; Sigma), and STI $(0.1 \mathrm{mg} / \mathrm{mL}$; Sigma) were used to eliminate Leydig cells. Collagenase IV $(0.1 \mathrm{mg} / \mathrm{mL}$; Sigma $)$ and DNase ( $5 \mu \mathrm{g} / \mathrm{mL}$; Sigma) were used to reduce peritubular cells. Then testicular cells were washed and plated in medium with gentamicin $(0.02 \mathrm{~g} / \mathrm{L}$; Sigma) and maintained in a humidified atmosphere at $34{ }^{\circ} \mathrm{C}$ with $5 \% \mathrm{CO}_{2}$ for $6-8 \mathrm{~h}$. Germ cells were harvested by shaking and suction gently ${ }^{41}$. The residual aggregate consisted of sertoli cells. For the subgroup meioitc prophase I spermatocytes, the method of STA-PUT was applied, and cells were isolated according to the diameters: leptotene, 8-10 $\mu \mathrm{m}$; zygotene, $10-12 \mu \mathrm{m}$; and pachytene, $14-18 \mu \mathrm{m}$.

\section{RNA isolation and real-time PCR}

Total RNA was extracted from the control and Uhrf1cKO mouse spermatocytes (leptotene/zygotene stage and pachytene stage) were isolated. The primary cells were homogenized in TRIzol reagent (Invitrogen), followed by RNA precipitation. cDNA was synthesized from $1 \mu \mathrm{g}$ RNA with a reverse transcription kit (TaKaRa). Real-time PCR was performed using SYBR Premier EX Taq (TaKaRa). Relative levels of mRNAs were normalized to the levels of endogenous $\beta$-Actin in the same samples. Genes were amplified with the specific primers (Table S1).

\section{Western blot}

Sixteen day post-partum (dpp) control and Uhrf1-cKO mouse tissue extracts containing $30 \mu \mathrm{g}$ proteins were resolved by sodium dodecyl sulfate-polyacrylamide gel electrophoresis and transferred to nitrocellulose (NC) membrane (Millipore Corp). After probing with primary antibodies, the membranes were incubated with secondary mouse or rabbit antibodies (1:2000). The primary antibodies used were UHRF1 (1:1000, Active motif, Cat\# 61341) and $\beta$-Actin (1:10,000, Abcam, Cat\# ab8227).

\section{Hydroxy-methylated DNA IP (hMeDIP)-qPCR}

Genomic DNA was extracted with the PureLink Genomic DNA Mini Kit (Thermo). Purified Genomic DNA was sonicated to an average size around $200 \mathrm{bp}$ (range: $100-500 \mathrm{bp}$ ) with a bioruptor (Diagenode). DNA fragments were end-repaired. A-tailed and custom TruSeq adapters were ligated using the TreSeq DNA sample preparation Kit (Illumina). The DNA fragments ligated with adapters were immunoprecipitated with Protein A+G magnetic beads coupled with $5 \mathrm{mC}$ or $5 \mathrm{hmC}$ antibody. The purified DNA samples were then ready for the qPCR test. The specific primers were listed in Table S1.

\section{Bisulfite sequencing}

Bisulfite conversion was performed using the EpiTect Bisulfite Kit (Qiagen) according to the manufacturer's protocol. Bisulfite-treated DNA was then used to amplify. The amplified regions were cloned into pEASYT1 (TransGen Biotech) and sequenced. The primers for PCR amplification were listed in Table S1.

\section{Dot blot assay}

Genomic DNA samples were heated at $95^{\circ} \mathrm{C}$ for $10 \mathrm{~min}$ and allowed to cool on ice, then the ice-cold $20 \times$ side scatter (SSC) was added. Meanwhile, an NC membrane and two filter papers were wetted with $6 \times$ SSC and then mounted on a 96-well dot blot apparatus. To the wells to be used, $500 \mu \mathrm{L}$ of water was added and pulled through the membrane with gentle vacuum pressure. Subsequently, the diluted samples were added and pulled through. The wells were then washed with $500 \mu \mathrm{L}$ of $2 \times$ SSC solution. The membrane was next allowed to air dry before ultraviolet DNA cross-linking for $5 \mathrm{~min}$ at $100 \mu \mathrm{J} /$ $\mathrm{cm}^{2}$. In all, $5 \%$ milk was applied for blocking for $1 \mathrm{~h}$, at room temperature. Then the membrane was incubated with $5 \mathrm{hmC}$ antibody (1:3000) for $3 \mathrm{~h}$ at room temperature. After three times washing with PBST (5 min each) and incubation with secondary antibody (1:2000) for $1 \mathrm{~h}$, the samples were developed with ECL. The density was calculated by the software of Image $1.52 \mathrm{a}$.

\section{Quantification and statistical analyses RNA-seq}

For RNA sequencing, four Uhrf1-cKO and control mice were sacrificed for isolating spermatocytes (leptotene/ zygotene and pachytene stages) by the method of STAPUT. To prevent cross-contamination, the leptotene/ zygotene stage spermatocytes were isolated from $12 \mathrm{dpp}$ mice and the pachytene stage spermatocytes were isolated 
from $16 \mathrm{dpp}$ mice. The indicated spermatocyte pools were then subjected to library construction. Libraries were prepared using NEB Next Ultra Directional RNA library preparation kit for Illumina. Quality control was carried out with a Bio-analyzer (Agilent), and 150-base-pair (bp) paired-ends sequencing was performed with a HiSeq $\mathrm{X}$-ten sequencer (Illumina). For each sample, the RNAseq data was mapped to mm10 genome by TopHat v2.0.7 with no more than two mismatches, and then only the uniquely mapped reads were used to estimate the expression values in gene level by RPKM (reads per kilobase of transcript, per million mapped reads) with featureCount (V1.5.3). Statistical significant test of differentially expressed genes (DEGs) was performed by DEGseq with R. Genes with absolute $\log _{2}$-transformed fold changes $>2$ were regarded as DEGs and a threshold of $p$ value $<0.001$ was used. DEGs were identified as significantly differential expression in either leptotene/ zygotene or pachytene stages. Hierarchical clustering of $\log _{2}$-transformed RPKMs was generated by Cluster 3.0 and visualized by Java TreeView. The raw next-generation sequencing (NGS) data were deposited to the NCBI SRA database under accession number (SRP201556). Some RNA sequencing results were verified by real-time PCR.

\section{MeDIP-seq and hMeDIP-seq}

The MeDIP and hMeDIP sequencing were performed as previously mentioned ${ }^{42,43}$. The meiotic prophase spermatocytes from 6 to 8 Uhrf1-cKO and control mice $(16 \mathrm{dpp})$ were prepared as aforementioned. The antibodies used for immunoprecipitation (IP) were $5 \mathrm{mC}$ and $5 \mathrm{hmC}$ (Active motif). Mouse or Rabbit IgGs were applied for nonspecific IP experiment as control samples. DNA libraries were generated using the NEB Next Ultra DNA library preparation kit for Illumina. Quality control was carried out with a Bioanalyzer (Agilent). Sequencing was performed on Illumina HiSeq X-ten sequencing platform. Sequencing reads were aligned to the reference genome (mm10) using Bowtie2 v2.3.3.1 with no more than two mismatches, and then only the uniquely mapped reads were used for peak calling analysis and mapping depth analysis. The mapping depth was normalized by the total mapped reads for each sequenced sample. The measurement of $5(\mathrm{~h}) \mathrm{mC}$ level (density) was only summarized with the normalized mapping depth of CpG sites in mouse reference genome (mm10). The peaks detection was performed by MACS V1.4.2 with default cut-off. Peaks were assigned to the nearest genes using Homer V4.8.2. The raw NGS data were deposited to the NCBI SRA database under accession number SRP201555.

\section{Statistical analysis}

All statistical data were analyzed with GraphPad Prism version 5 . The statistical data of litter size, testis weight, numbers of spermatocytes with the indicated meiotic biomarkers, and RT-PCRs were presented as means \pm SEM. Analysis of variance or Student's $t$ test were used for statistical comparison to determine significance. Statistical significance was set as: NS, $p>0.05$; ${ }^{*} p \leq 0.05$; ${ }^{* * *} p \leq$ 0.01 ; ${ }^{* * * * *} p \leq 0.001$. All presented results were from at least three independent experiments. The investigators were blinded to the group allocation during the experiment and when assessing the outcome.

\section{Results}

\section{The distribution of UHRF1 in male germ cells}

The initial experiments were aimed to examine the expression pattern of UHRF1 in germ cells across the male meiotic prophase I utilizing an IHC assay. The UHRF1 protein was detected within the entire nuclei in spermatogonia (arrowed in red) and preleptonema/leptonema (arrowed in green) but started to concentrate on the chromosomes from zygotene stage (Fig. 1a and enlargement). Note that the enlargements 1 and 2 showed the expression of UHRF1 in the zygonema and pachynema/diplonema, respectively. Next we analyzed the precise location of UHRF1 in testicular spreads. Figure 1b showed an alteration of UHRF1 expression from leptonema to diplonema. The expression of UHRF1 was scattered in leptonema and zygonema but condensed in pachynema and diplonema. The shift of the UHRF1 expression pattern from a random to condensed appearance implied that Uhrf1 exerted different physiological roles in the murine prophase meiosis I.

\section{Ablation of UHRF1 in germ cells caused male sterility with impaired testis development}

To analyze the physiological role of UHRF1 in male meiosis, a conditional knockout mouse strain was established. The Stra8-cre tool mouse was used to delete the floxed exon4 of Uhrf1 (Fig. 1c, d). Western blot assay showed that the level of UHRF1 was reduced apparently in the Uhrfi ${ }^{f / f}$;Stra8-cre mouse testicle tissue. IHC analysis showed the dramatic loss of UHRF1 in the Uhrfi fff; Stra8-cre mouse germ cells compared with the Uhrff fif germ cells (Fig. 1e, f). These results indicated that UHRF1 was deleted efficiently in germ cells.

The UHRF1-deficient male mice were apparently normal in growth (data not shown) but infertile. The mutant adult mice were lacking spermatozoa and had reduced size testes (Fig. 2a, b). To validate the testicular defects, we examined the weight gain between the Uhrff ${ }^{\text {fff }}$;Stra8-cre and control mice at $7,10,13,18,24$, and $36 \mathrm{dpp}$,

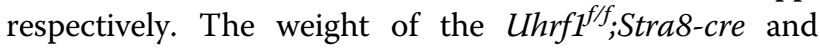
control groups showed no apparent difference from 7 to $13 \mathrm{dpp}$. As the growth continued, the mean testicle weight of the Uhrf1 $1^{f / f}$;Stra8-cre group were significantly lower than that in the control group at each indicated time 
A

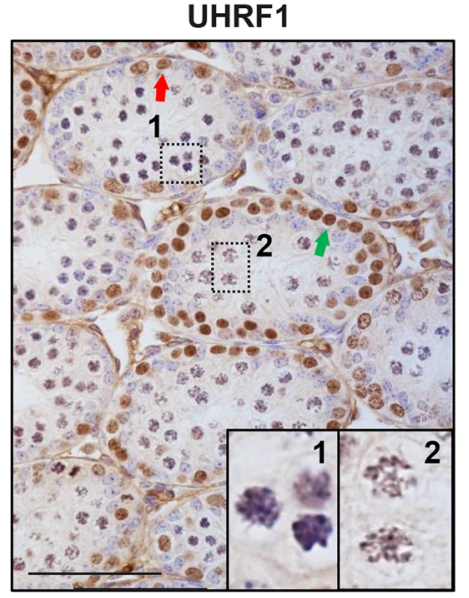

B
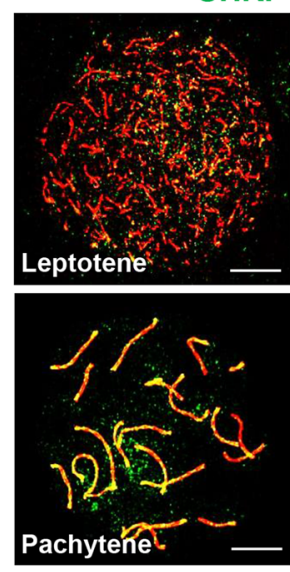

UHRF1/SYCP3
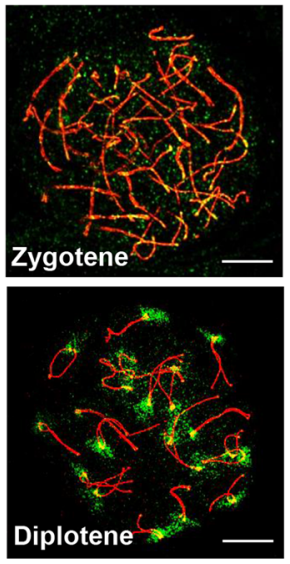

C

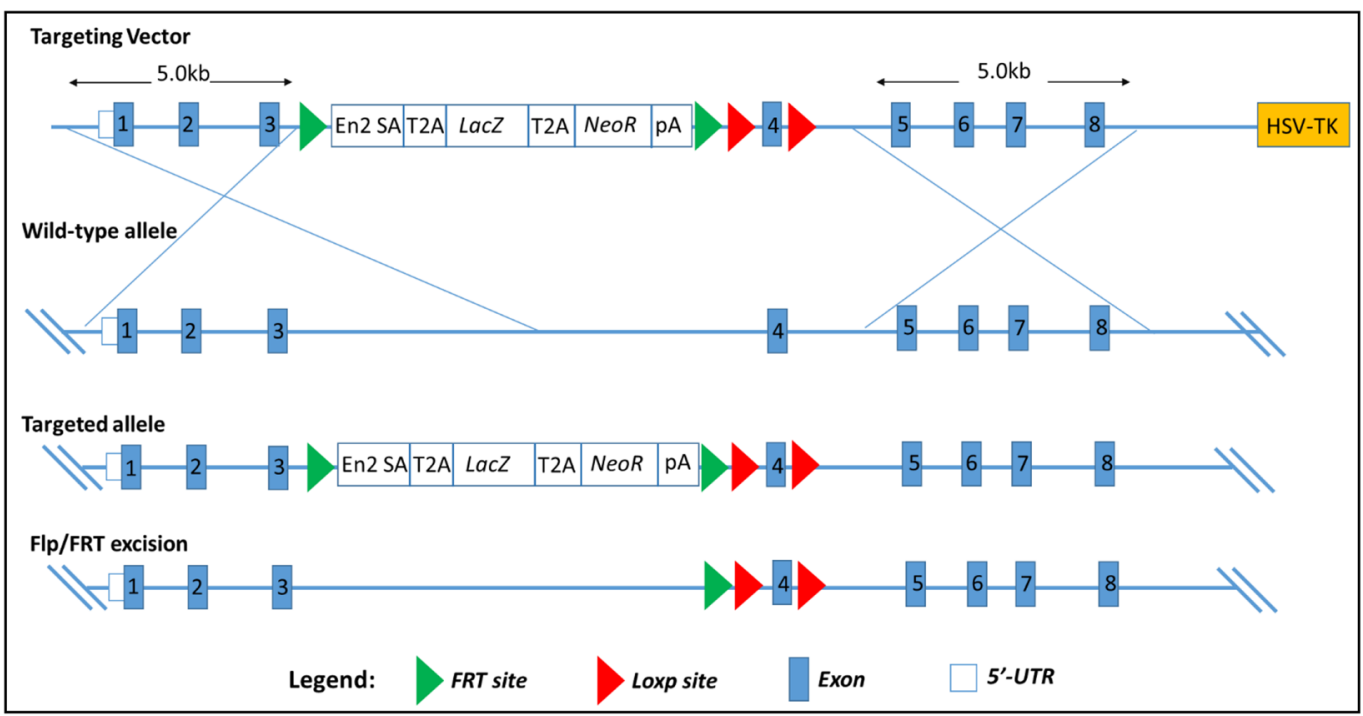

D

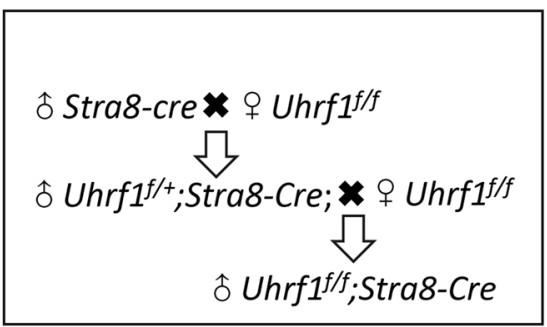

E

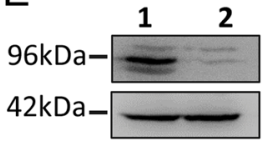

UHRF1

1 Uhrf1 f/f

$\beta$-Actin

2 Uhrfiff/f; Stra8-cre

F
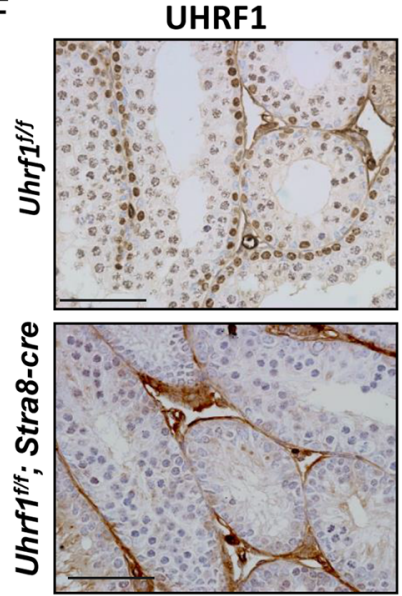

Fig. 1 UHRF1 was expressed in the meiotic prophase I and the gene-trapped Uhrf1 conditional knockout mouse was prepared. a The expression of UHRF1 protein in the mouse testicular tissue. $\mathbf{b}$ The distribution of UHRF1 protein (green) in the sub-stages of mouse meiotic prophase. c Schematic diagram of lox P-deletion system in the Uhrf1 gene in mouse; $\mathbf{d}$ mouse strategy; $\mathbf{e}$, $\mathbf{f}$ western blot and immunohistochemical assay to the $16 \mathrm{dpp}$ mouse testicle tissue showing the knockout efficiency of UHRF1 protein. At least three independent experiments were carried out. Scale bar, $25 \mu \mathrm{m}$ in $\mathbf{a}, \mathbf{f}, 5 \mu \mathrm{m}$ in $\mathbf{b}$ 

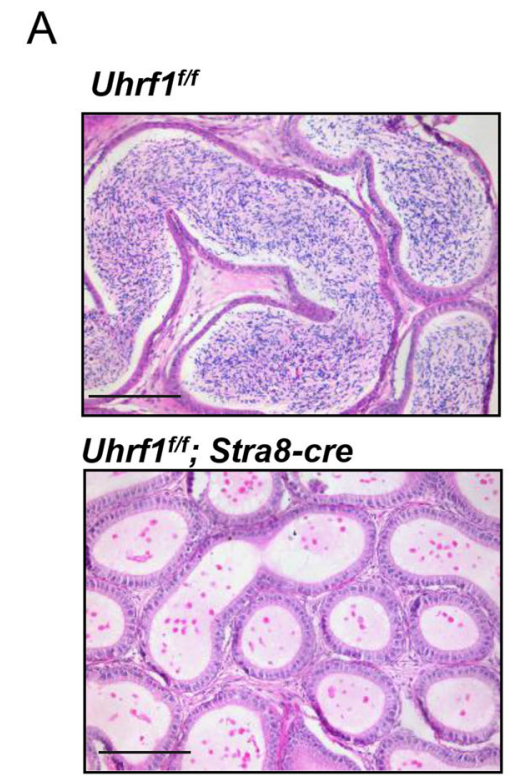

C
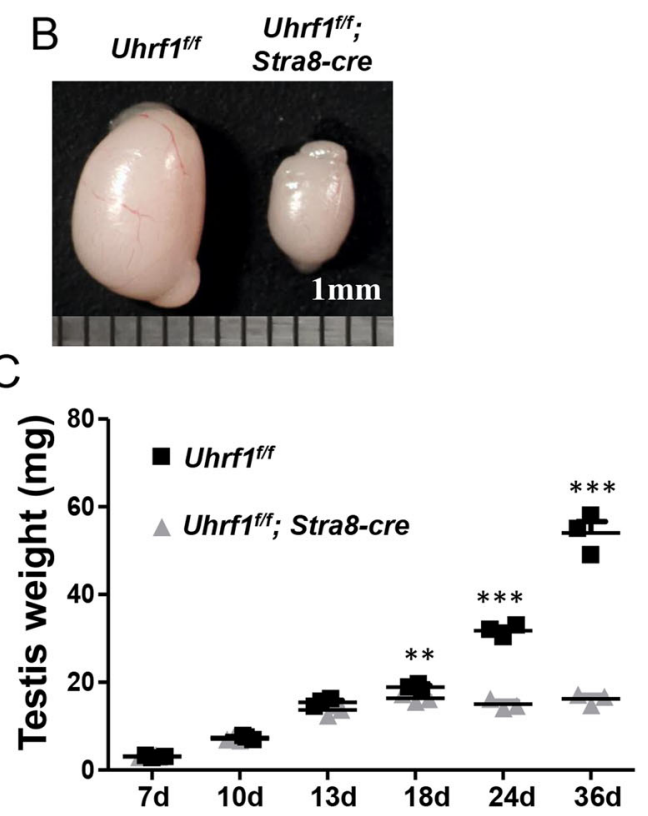
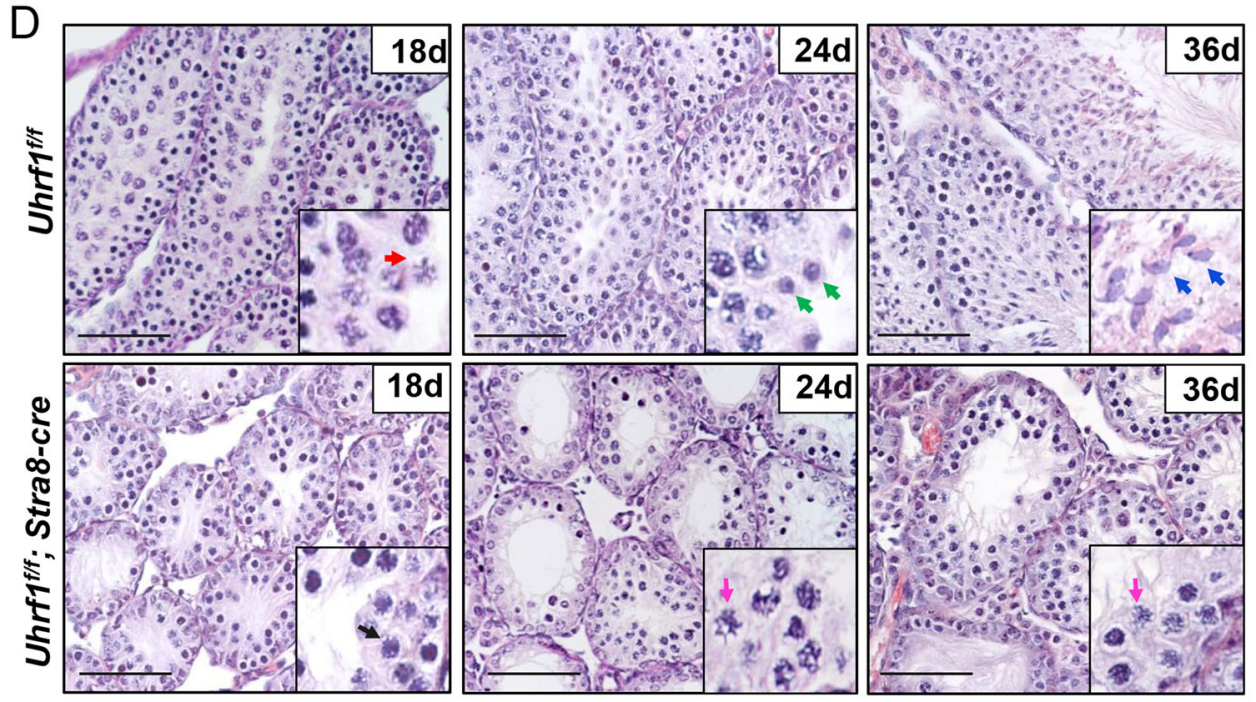

$\mathrm{E}$

Uhrfiff

Uhrf1ff; Stra8-cre
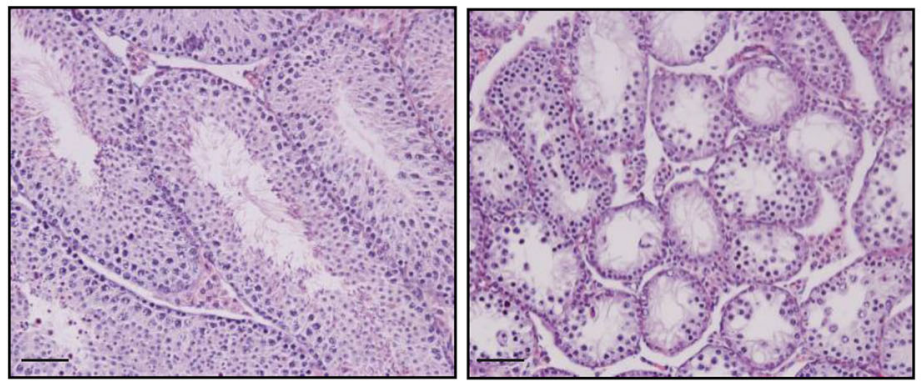

Fig. 2 UHRF1 was required for the mouse spermatogenesis and fertility. a H\&E staining of epididymal sections from 12-week-old Uhrf $1^{\mathrm{ff} / \text {; }}$;tra8cre and Uhrff ${ }^{f / f}$ mice. b Morphology of testes. c The mean testicular weights of the mice from 7 to $36 \mathrm{dpp}$. $\mathbf{d}$ H\&E staining of testicular sections at 18 , 24, and $36 \mathrm{dpp}$. e Histology assay showing the testicular tubes in the adult Uhrf ${ }^{\mathrm{f} / f}$;Stra8-cre and Uhrf f $^{\mathrm{f} / \mathrm{f}}$ sections. Scale bar, $25 \mu \mathrm{m}$. dpp day postpartum. At least three independent experiments were carried out, data are presented as mean \pm SEM of three mice in $\mathbf{c}$. ${ }^{* *} p \leq 0.001 ;{ }^{* *} p \leq 0.01 ; N S$, $p>0.05$. 
points (Uhrff fff: $18.9 \pm 0.7 \mathrm{mg}(18 \mathrm{dpp}), 31.8 \pm 1.3 \mathrm{mg}(24$ dpp), $54.0 \pm 4.5 \mathrm{mg}$ (36 dpp); Uhrff ${ }^{f / f}$;Stra8-cre: $16.4 \pm$ $0.9 \mathrm{mg}$ (18 dpp), $15.1 \pm 1.0 \mathrm{mg}$ ( $24 \mathrm{dpp}$ ), $16.3 \pm 1.2 \mathrm{mg}$ (36 dpp); $n=3$; Fig. 2c). These results suggested that loss of UHRF1 led to a failure of testicle development.

The development of the Uhrf1 knockout mouse germ cell was apparently normal shortly after entering into the meiosis. An ordered arrangement of preleptotene, leptotene, zygotene, and early pachytene stage spermatocytes was observed both in the Uhrf $1^{f f f}$;Stra8-cre and Uhrf1 fff groups at 7 and $12 \mathrm{dpp}$ (data not shown). Figure $2 \mathrm{~d}$ showed the middle/late pachytene spermatocytes with the characteristic of thick fibers separated within the nucleus and obvious sex bodies (arrowed in red) in the control sections. However, the spermatocytes were still in the early pachytene-like stage with the characteristic of heavily stained thick fibers but absent sex bodies in the mutant mouse testis sections at $18 \mathrm{dpp}$ (arrowed in black). At 24 and $36 \mathrm{dpp}$, we detected the haploid spermatids (arrowed in green) and spermatozoa (arrowed in blue) in the control testes. However, the most advanced stage of spermatocyte was still at the early pachytene-like stage (arrowed in pink) in the Uhrf1 $\mathrm{f}^{\mathrm{ff}}$;Stra8-cre mouse sections without spermatids or spermatozoa. These histological defects implied that the impairment of spermatocyte development caused by the deletion of UHRF1 occurred in the process of meiosis I. In adult, we detected that the Uhrf1 conditional knockout testes were impaired even severely with fewer spermatogonium or early stage spermatocytes and were also devoid of spermatids (Fig. 2e). We assumed this might probably be due to the first expression of Stra8-cre in differentiating spermatogonia and the age effect of spermatogonia.

\section{Loss of UHRF1 resulted in a disturbed meiotic prophase progression, impaired SC assembly, and affected homology synapsis}

For further analysis, we examined spermatocyte spreads from 8-week-old mice. The ratios of the leptotene and zygotene stage cells of the Uhrff fff; Stra8-cre mice were increased significantly compared with the control group ( $\sim 33.3 \%$ vs $\sim 11.0 \%, \sim 45.6 \%$ vs $\sim 12.0 \%)$, while the ratios of pachytene and diplotene stage cells were decreased in the Uhrf ${ }^{f / f}$; Stra8-cre mice ( $16.4 \%$ vs $\sim 61.7 \%, \sim 2.2 \%$ vs $\sim 15.3 \%$ ) (Fig. 3a). This data suggested that UHRF1 deficiency interfered severely with the progression of meiotic prophase.

The SC is formed by two lateral elements (LEs), between which the transverse filaments (SYCP1) spanned. The thread-like SYCP3, a major composition of LE, was observed in the control spermatocytes, whereas a large number of spot-like distribution pattern of SYCP3 was found in the mutant germ cells (Fig. 3b). The leptonema and zygonema without UHRF1 were preferentially associated with defected SYCP3 stretches (Fig. 3c, d). These aberrant localizations of SYCP3 (arrowed in red) indicated the impaired assembly of LEs. In the pachytenelike stage, we found the failure of homologous synapsis in some chromosomes (arrowed in yellow and enlargement) in the mutant spermatocytes. Together with the dramatically reduced pachynema number, these above observations suggested that UHRF1 deficiency led to the impaired assembly of axial/lateral elements (AE/LEs), which resulted in the failure of SC formation. The abnormal distribution of SYCP1 verified such SC assembly defect (Fig. 3e, f).

\section{UHRF1 was essential for the meiotic recombination and pachytene development}

Meiotic recombination is tightly coupled with synapsis $^{44}$, so we determined whether the deficiency of UHRF1 impaired meiotic recombination. Following the DNA double-strand break (DSB) formation, the DNA ends are engaged in a process of maturation. The DMC1 complex is recruited to the $3^{\prime}$ single-strand DNA tails to promote homology search and strand exchange in the zygotene stage. After the finish of DSB repair, most of the DMC1 are released from the resolved DSBs in pachynema. Recombination shuffles parental genomes through genetic exchanges leading to crossovers (COs) or non-crossovers $(\mathrm{NCOs})^{45}$. The NCOs have only a limited and local effect on genetic diversity, while COs exert critical roles in ensuring an accurate segregation of homologs and generating genetic diversity ${ }^{44}$. The presence of MLH1 sites on paired chromosomes is the final marker for sites that will result in $\mathrm{COs}^{46}$. Here the DMC1 foci were increased both in the zygotene and pachytene stages. The excessive DMC1 foci suggested that loss of UHRF1 led to an increased number of DSB sites in the Uhrff fff; Stra8-cre zygonema. And the retention of DMC1 foci implying the unresolved DSBs suggested that loss of UHRF1 led to DSB repair deficiency (Fig. $4 \mathrm{a}-\mathrm{c}$ ). This deficient DSB repair was further confirmed by the $\gamma \mathrm{H} 2 \mathrm{AX}$ defect (a histone variant $\mathrm{H} 2 \mathrm{AX}$ with phosphor-Ser139, restricted to the DSB foci; Fig. 4d, e). Only the sex body (white dashed regions, left) was stained with $\gamma \mathrm{H} 2 \mathrm{AX}$ antibody in the control pachynema, indicating that most DSBs were repaired in the autosomes. However, the UHRF1-deficient pachynema were with impaired sex body (white dashed regions, right). $\gamma \mathrm{H} 2 \mathrm{AX}$ foci remaining on the autosomes (arrowed in white) indicated the presence of un-repaired DSBs. A reduced number of MLH1 foci was also detected in the mutant pachynema (Fig. 4f, g) showing that loss of UHRF1 also resulted in impaired CO. The failure of H1T staining, a known mid/late pachytene marker ${ }^{47}$, further demonstrated the defective pachynema in the mutant mice (Fig. 4h, i). 


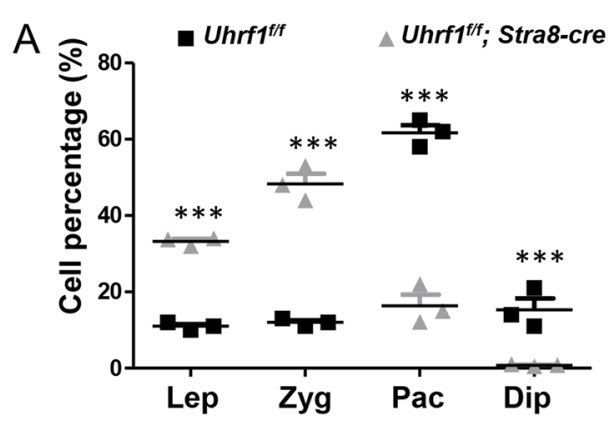

B
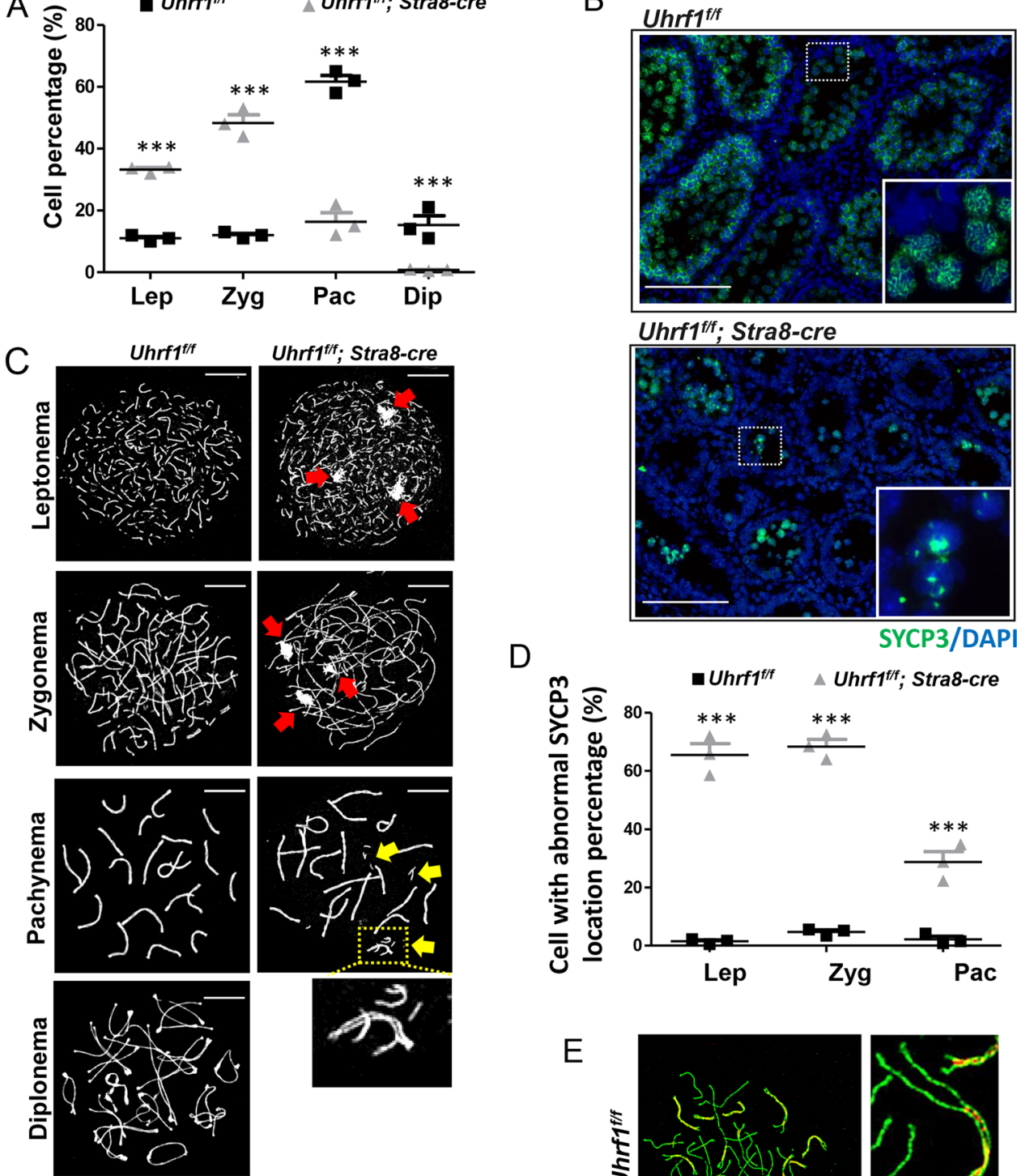

D
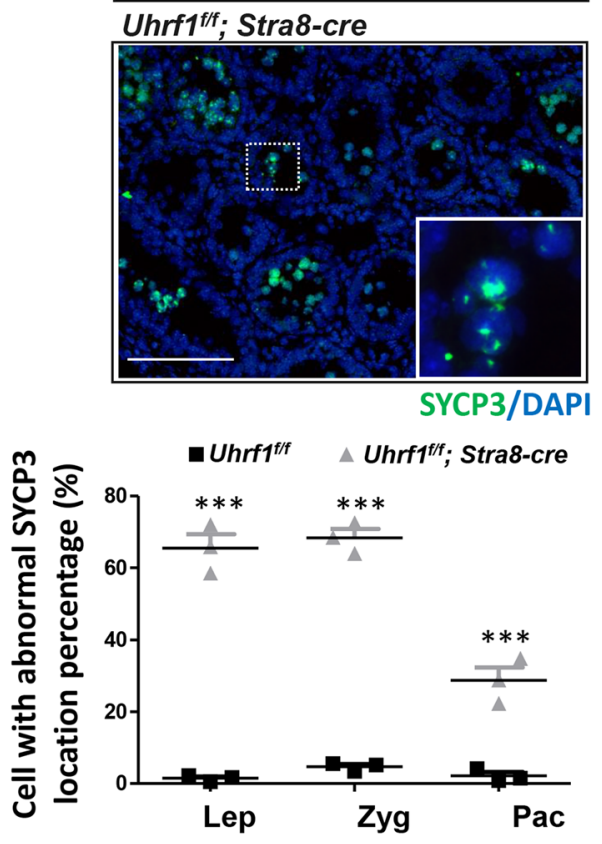

$\mathrm{F}$

- Uhrf1/f $\quad$ Uhrf1/fif; Stra8-cre

E
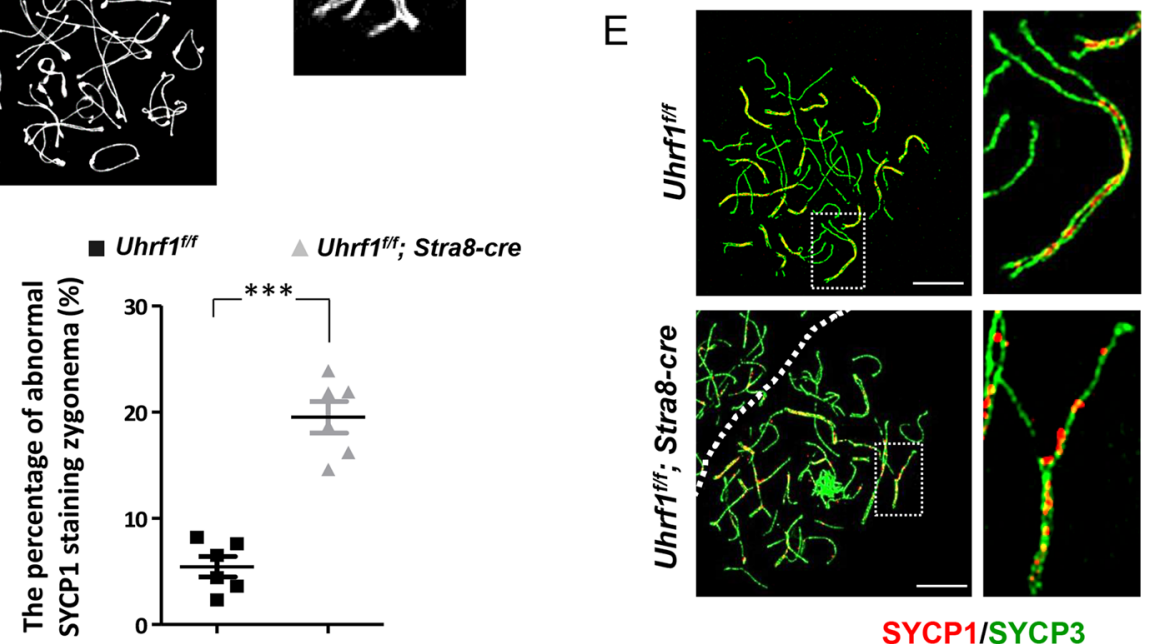

SYCP1/SYCP3

Fig. 3 UHRF1 deletion disrupted the meiotic progression and synaptonemal complex assembly. a Relative amounts of four spermatocyte populations (leptotene stage, zygotene stage, pachytene stage, and diplotene stage) during the prophase I in testes based on analyzing $>600$ spermatocytes in each stage. $\mathbf{b}, \mathbf{c}$ The immunostaining of SYCP3 in the testicular sections (b) and surface-spread chromatin preparations of Uhrf1 deletion and control mice (c); $\mathbf{d}$ the percentage of spermatocytes with abnormal SYCP3 location. e Double immunofluorescence of testicular spread preparations of the adult mice, SYCP3 (green) and SYCP1 (red). $\mathbf{f}$ The percentage of spermatocytes with abnormal SYCP1 location. Lep leptotene, Zyg zygotene, Pac pachytene, Dip diplotene. Data are presented as mean \pm SEM of three mice. ${ }^{* * *} p \leq 0.001$. Scale bar, $25 \mu \mathrm{m}$ in $\mathbf{b}$, $5 \mu \mathrm{m}$ in $\mathbf{c}$, e. 

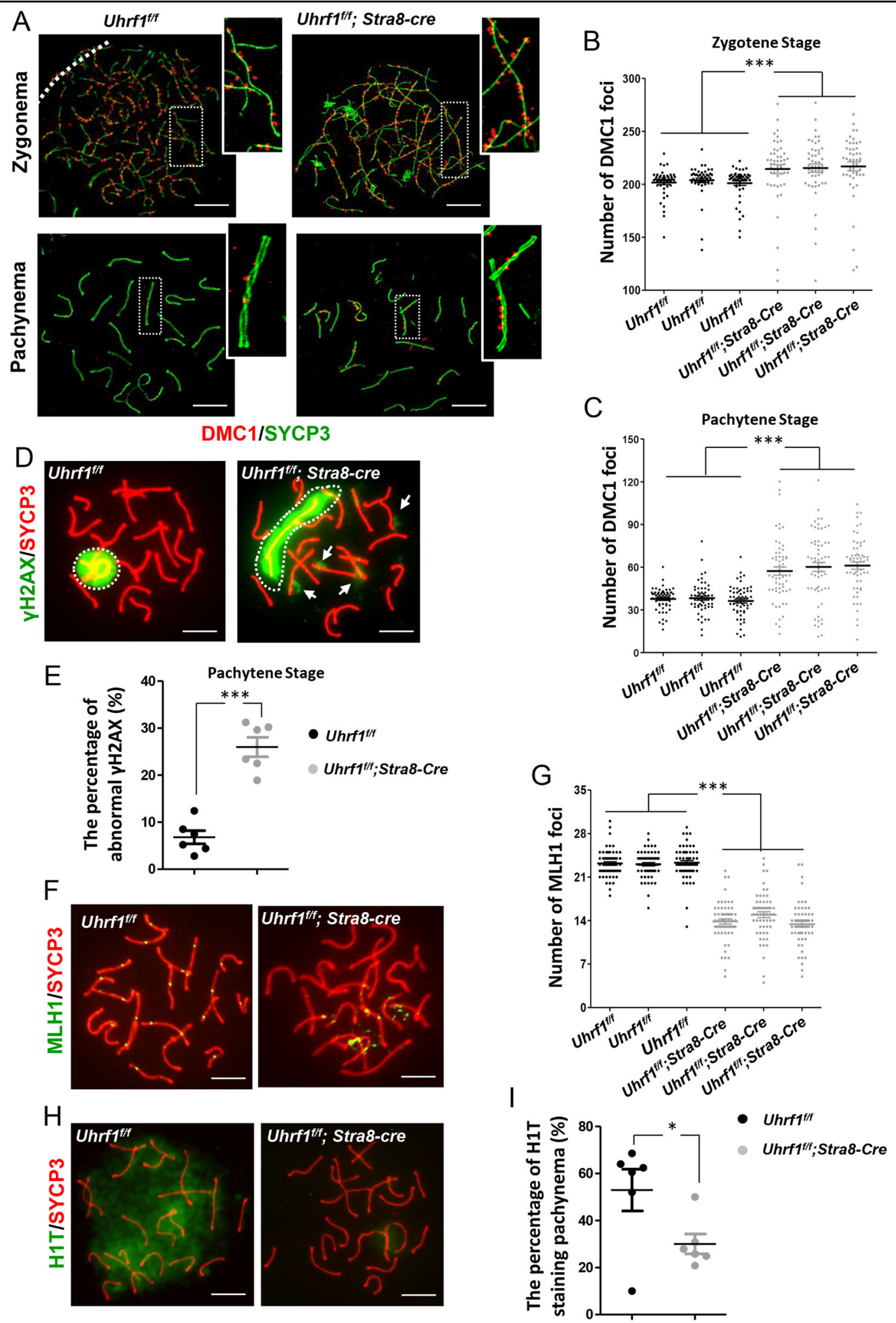

Fig. 4 UHRF1 deficiency resulted in impaired meiotic recombination and defective pachynema. a Double immunofluorescence of SYCP3 (green) and DMC1 (red) in testicular spread preparations. b, c The number of DMC1 foci in zygotene stage (b) and pachytene stage (c). d Immunostaining for SYCP3 (red) and YH2AX (green). e The percentage of abnormal $\gamma \mathrm{H} 2 \mathrm{AX}$ foci in the pachytene stage. $\mathbf{f}$ Immunostaining for SYCP3 (red) and MLH1 (green). $\mathbf{g}$ The number of MLH1 foci in pachynema. $\mathbf{h}$ Immunostaining for SYCP3 (red) and H1t (green). $\mathbf{i}$ The percentage of spermatocytes with H1T staining. ${ }^{* *} p \leq 0.001 ;{ }^{*} p \leq 0.05$. Scale bar, $5 \mu \mathrm{m}$ in $\mathbf{a}, \mathbf{d}, \mathbf{f}, \mathbf{h}$. 
Collectively, deletion of UHRF1 caused a skewed recombination profile with the characteristic of excessive and retained early recombination foci but depleted the late foci and a poor incorporation of histone $\mathrm{H} 1 \mathrm{~T}$ into meiotic chromatin.

\section{Knockout of UHRF1 altered the gene expression and led to global hypomethylation}

To identify DEGs in the UHRF1-deficient spermatocytes that impair the progression of meiotic prophase, we performed RNA-seq and qPCR to isolate spermatocytes from Uhrfi $1^{f / f}$;Stra8-cre and Uhrff ${ }^{f / f}$ mouse (leptotene/ zygotene and pachytene stages). A total number of 4768 genes were downregulated and 981 genes were upregulated with $p$ value $<0.001, \mid \log _{2}$ fold change $\mid>1$ in either of these two stages (Fig. 5a, b and Table S2). Gene Ontology (Cluster Profiler V3.4.4) highlighted the that DEGs were involved in the regulation of the meiosis process (Fig. 5c). Many meiosis-associated genes were altered. The downregulation of SC assembly gene $E h m t 2^{48}$, recombinationassociated gene $\mathrm{Setx} \mathrm{x}^{49}$ and $\mathrm{Ndrg} 3^{50}$, chromosome segregation gene $S m c 5^{51,52}$, and $H 1 t$ transcriptional regulator $R f \times 2^{53}$ provided the molecular mechanisms by which how deficiency of UHRF1 generated the defects in SC assembly, recombination, and $\mathrm{H} 1 \mathrm{t}$ incorporation (Fig. 5d). Interestingly, we detected an increase of Syce $3^{54,55}$, which was specifically located in the central element in the leptotene/zygotene stage. The increase of Syce 3 as well as the inter-sister chromosome distribution of SYCP1 (Fig. 3e) further confirmed the SC assembly defect.

Considering that UHRF1 is critical to DNA methylation (DNAme), we examined the genomic level of DNAme. The methylated DNA immunoprecipitation-sequencing (MeDIP-seq) showed an average loss of DNAme approximately by $15.83 \%$ in genome in the Uhrf $\mathrm{f}^{\mathrm{ff} \text {; }}$ Stra 8 -cre spermatocytes (Fig. 5e). A validation was performed by the bisulfite assay to some retrotransposable elements, which were reported to be hyper-methylated ${ }^{56,57}$ (Supplementary Fig. S1). The methylation rates of IAPEz, L1Md_T, and RTRL8-int were $88.19 \%, 88.39 \%$, and $88.07 \%$ in the Uhrf $f^{f / f}$ meiotic prophase cells, while the rates were decreased to $25.69 \%, 61.16 \%$, and $40.34 \%$ in the Uhrf $\mathrm{f}^{\mathrm{ff} f}$;Stra8-cre cells, respectively. Given that the $5 \mathrm{mC}$ enrichment in the promoter regions is negatively correlated to the transcriptional level $^{58}$, we compared the results from MeDIP-seq and RNA-seq (upregulated genes). Figure $5 f$ showed that the DNA hypo-5mC in the promoters had a limited effect on the transcriptional level. Only $16(\sim 1.63 \%)$ genes, most of which were without clear function in meiosis, were found to have hypomethylation levels in their promoter regions in the Uhrf1 $\mathrm{f}^{f / f}$;Stra8-cre spermatocytes. The little effect on gene transcription of hypomethylation in the Uhrf 1 knockout mice implied an unexplained mechanism of UHRF1 on the regulation of meiotic gene expression.

\section{Blocking of UHRF1 exhibited hyper-5hmC in the TSS region of downregulated genes}

During the meiotic prophase I, the global DNAme maintains constantly, whereas the $5 \mathrm{hmC}$ reduces dramatically ${ }^{22,59}$. There were 12,083 genes reported with $5 \mathrm{hmC}$ downregulated from preleptonema to pachynema ${ }^{22}$. As the distribution of $5 \mathrm{hmC}$ is highly associated with gene expression, we compared our RNA-seq data (significantly downregulated genes) with this reported set of 12,083 genes and found $>65.73 \%$ of the repressed genes in the UHRF1-deficient spermatocytes were overlapped (Fig. 6a). We assumed that the deficiency of UHRF1 led to an altered $5 \mathrm{hmC}$. To verify this assumption, we checked the global $5 \mathrm{hmC}$ in the Uhrff ${ }^{f f f}$;Stra8-cre and Uhrff $\mathrm{f}^{f / f}$ mouse meiotic prophase cells. Dot blot assay revealed that the deficiency of UHRF1 resulted in an upregulation of global DNA 5hmC (Fig. 6b). For further analysis, the hMeDIPseq was carried out. hMeDIP-seq showed UHRF1 deficiency led to a dysregulated DNA hyper-5hmC in all chromosomes (Fig. 6c). 4789 hMeDIP peaks were detected in the mutant spermatocytes, whereas the number in the control was 3836 . Both of the hMeDIP peaks were diversified in distribution at promoter, $5^{\prime}$ or $3^{\prime}$ UTR, and exon and intron or intragenic regions (Fig. 6d). However, it would be noteworthy that there were 3668 peaks unique in the mutant cells, suggesting the $5 \mathrm{hmC}$ profile was greatly changed without UHRF1 (Fig. 6e).

The $5 \mathrm{hmC}$ distribution in the promoter region and gene body contributes to gene repression and activation, respectively ${ }^{60,61}$. We mapped the hMeDIP peaks of DEGs, finding the average level of $5 \mathrm{hmC}$ raised by $16.29 \%$, $13.30 \%$, and $13.34 \%$ significantly in the regions of proximal promoter ( -5 to $-0.1 \mathrm{~kb}$ from TSS), TSS $( \pm 0.1 \mathrm{~kb}$ from TSS), and gene body ( $0.1 \mathrm{~kb}$ from TSS), respectively, in the downregulated gene set. In the upregulated gene set, the average level of $5 \mathrm{hmC}$ rate raised by $16.33 \%$ significantly in the region of proximal promoter. However, the changes of average level of $5 \mathrm{hmC}$ rate in the regions of TSS and gene body was not statistically significant $(p=$ 0.402 and $p=0.239$, respectively, Student's $t$ test; Fig. 6 f). A previous report suggested that the inhibition of transcriptional activity is primarily due to the presence of $5 \mathrm{hmC}$ in the promoter and that $5 \mathrm{hmC}$ in the gene body has a minimal effect on transcription ${ }^{61}$. Here our result showing the elevated $5 \mathrm{hmC}$ level in TSS was highly associated with downregulated DEGs implied the primary repressing role of $5 \mathrm{hmC}$ in TSS in gene transcription. However, the upregulated genes seemed to be resistant to this repressive effect of UHRF1 to $5 \mathrm{hmC}$ around TSS, because the upregulated genes failed to raise much $5 \mathrm{hmC}$ rate significantly. UHRF1 deletion might increase their expression by other mechanisms, considering it a multiple functional domain-containing protein. Furthermore, we found that the refgenes' peaks of $5 \mathrm{hmC}$ in TSS region 


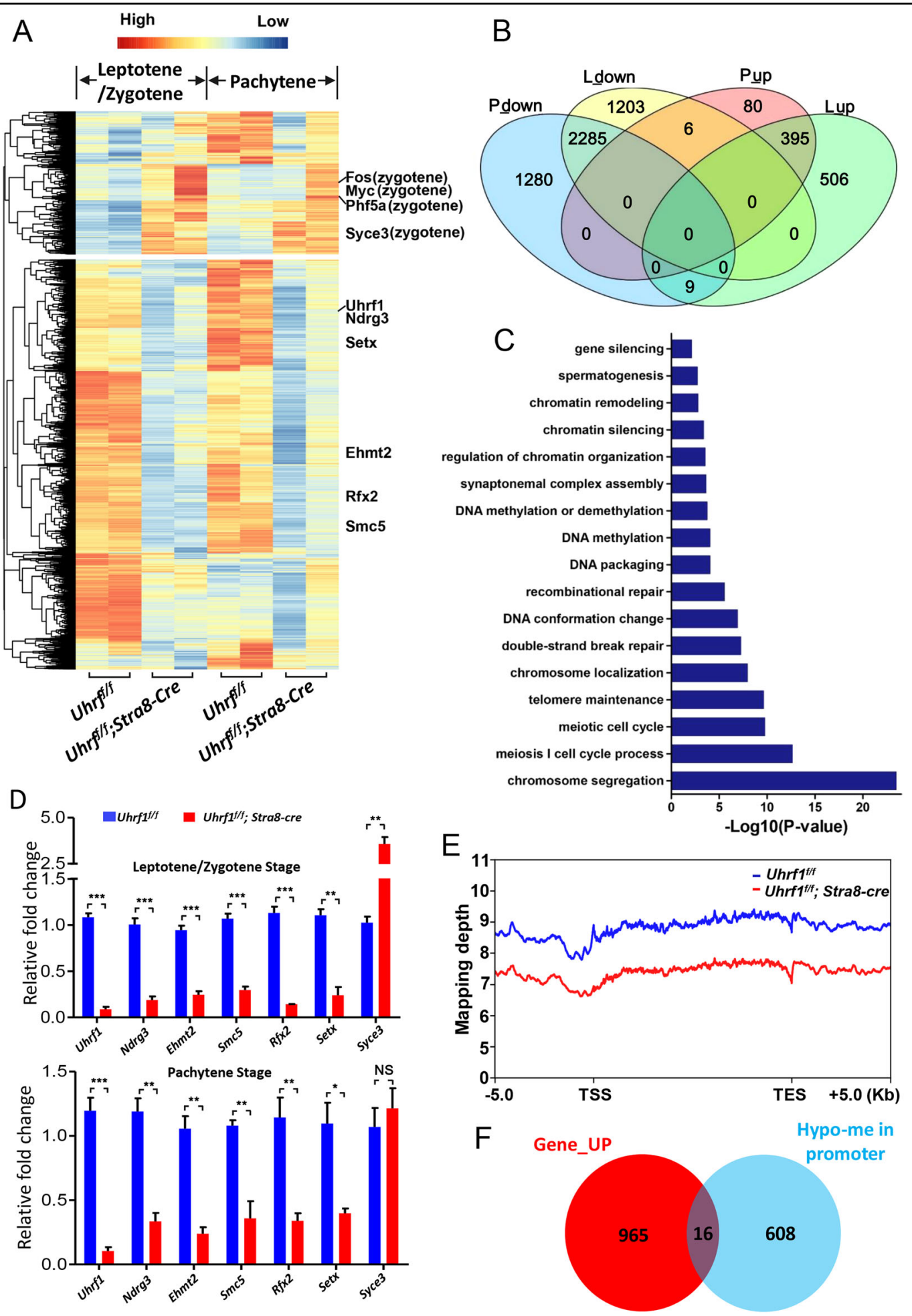

Fig. 5 The gene expression and global methylation after UHRF1 deletion. a RNA-seq for mouse spermatocytes (leptotene/zygotene and pachytene stages). Clustered heat map of $\log _{2}$-transformed RPKMs showing the differentially expressed genes after UHRF1 deletion. Indicated genes are marked in right. $\mathbf{b}$ Identified differentially expressed genes were highly consistent between leptotene/zygotene and pachytene stages. (P pachytene stage genes, L leptotene/zygotene stage genes). c Gene ontology analysis of DEGs using the database of ClusterProfiler V3.4.4. d RT-PCR assay showing the relative change of some representative meiotic genes. e DNAme densities across the gene bodies of all reference genes. $\mathbf{f}$ Venn diagram depicting a set of 16 genes that were induced in transcripts and hypomethylated in promoter and $5^{\prime}$ UTR regions (unique in control) from the leptotene to pachytene stage in the UHRF1-deficient spermatocytes. For the upregulated transcripts, the cut-off was $p$ value $<0.001$ with fold change $>2$. 


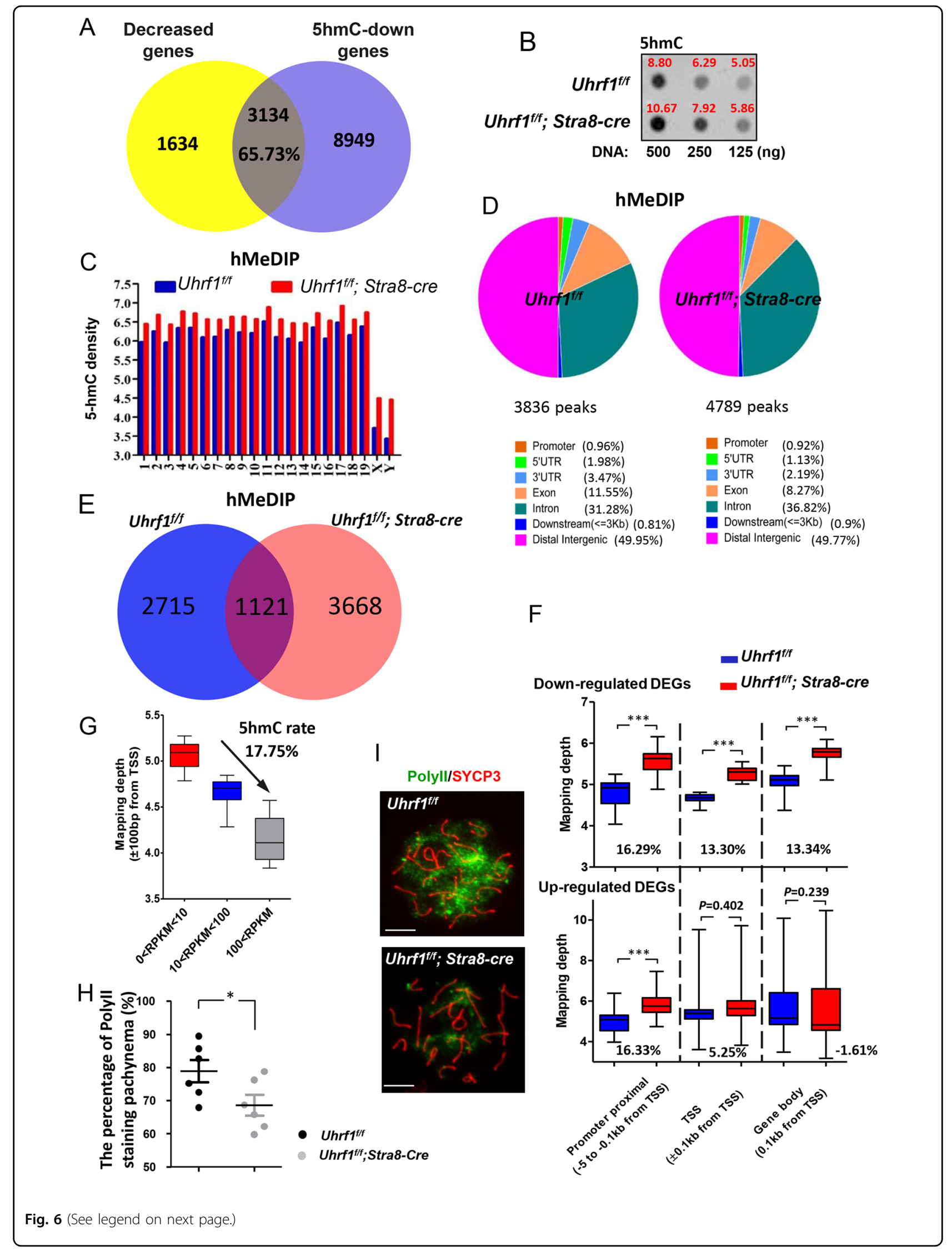


(see figure on previous page)

Fig. 6 Hyper-5hmC resulted from UHRF1 deletion. a Venn diagram depicting reduced transcripts associated with $5 \mathrm{hmC}$ downregulation. $\mathbf{b} 5 \mathrm{hmC}$ level of meiosis prophase I spermatocytes ( $16 \mathrm{dpp})$. c $5 \mathrm{hmC}$ densities of all chromosomes. $\mathbf{d}$ The distribution of $5 \mathrm{hmC}$ density on the genome of spermatocytes. e Venn diagram depicting $5 \mathrm{hmC}$ peaks in Uhrff ${ }^{f / f}$;Stra8-cre and Uhrff ${ }^{\mathrm{f} / \mathrm{f}}$ spermatocytes. $\mathrm{f} 5 \mathrm{hmC}$ densities was shown in the proximal promoter, TSS, and gene body regions of the DEGs. $\mathbf{g} 5 \mathrm{hmC}$ densities in TSSs of the total refgenes with different RPKMs. $\mathbf{h}$ The percentage of RNA polymerase II staining in pachynema. i Double immunofluorescence of testicular spread preparations, SYCP3 (red) and RNA polymerase II (green). Scale bar, $5 \mu \mathrm{m}$ in i.

were negatively associated with RPKMs. The $5 \mathrm{hmC}$ in TSS region was decreased by $17.75 \%$ from the low RPKM gene set $(0<$ RPKM $<10)$ to high RPKM gene set $($ RPKM $>$ 100) (Fig. 6g). To validate, we examined the $5 \mathrm{hmC}$ level in TSS region of some decreased meiotic genes such as $\operatorname{Dazap1}^{62}$, Ehmt $2^{48}, \operatorname{Rifl}^{63}$, and $R a d 23^{64}$ using the hMeDIP-qPCR ${ }^{65}$ and EpiMark 5-hmC and 5-mC Analysis Kit (NEB, E3317S), which was applied to determine the $5 \mathrm{hmC}$ rate by us and others previously ${ }^{66,67}$. The consensus results further confirmed that the deficiency of UHRF1 exhibited hyper-5hmC in the TSS region in the downregulated genes (Supplementary Fig. S2).

$5 \mathrm{hmC}$ at TSS inhibits the binding of RNA-Pol2 to DNA $^{60,61,68}$. Here a diminished RNA-Pol2 in the UHRF1deleted spermatocytes probably provided a piece of evidence that the hyper-5hmC in the repressed gene TSS region could possibly contribute to the affected RNA-Pol2 loading to decrease gene transcription. The average percentage of RNA-Pol 2 staining was $78.9 \%$ in the control spermatocytes, while the ratio was decreased significantly to 68.6\% in the UHRF1-deficient spermatocytes (Fig. 6h). In some Uhrf1 knockout spermatocytes, a severe loss of RNAPol2 signal was found (Fig. 6i). The different degrees of the impairment of RNA-Pol2 staining implied that there might be other potential mechanisms that UHRF1 regulated RNA-Pol2 binding besides the $5 \mathrm{hmC}$-TSS-dependent way.

\section{The levels of $5 \mathrm{hmC}$ and TET1 were upregulated after hypo- methylation in the UHRF1-deleted spermatocyte}

Considering the presence of hyper- $5 \mathrm{hmC}$ caused by the deletion of UHRF1, we assumed UHRF1 repressed 5hmC in spermatocytes. UHRF1 harbors multiple domains ${ }^{69}$. For example, the Tandem Tudor domain (TTD) allows UHRF1 to bind to di-/tri-methylated H3K9, the Set and RING Associated (SRA) domain facilitates in maintaining DNA methylation and histone modifications by recruiting DNMT1 and HDAC1, and Really Interesting New Gene (RING) domain confers intrinsic E3 ligase activity toward histones and non-histone proteins. Thus we next determined which domain was critical in this repression role. In vitro assay showed that a weak level of $5 \mathrm{hmC}$ signal was observed in approximately $65.1 \%$ and $64.27 \%$ GC-1 cells transiently transfected with full UHRF1 (pcDNA3.1UHRF1-Flag) and mutant UHRF1 with TTD domain deleted (pcDNA3.1- ${ }_{m}$ UHRF1- $\triangle$ TTD-Flag) plasmids, respectively. However, when the cells overexpressed the mutant UHRF1 with SRA domain deleted (pcDNA3. 1- ${ }_{m}$ UHRF1- $\triangle S R A$-Flag) and mutant UHRF1 with RING domain deleted (pcDNA3.1- UHRF1-DRING-Flag) plasmids, $73.1 \%$ and $61.83 \%$ GC-1 cells were with strong $5 \mathrm{hmC}$ signals, respectively (Fig. 7a, b). These results indicated that SRA and RING domains were indispensable for UHRF1-repressed global DNA 5'hydoxymethylation.

As we observed that DNA 5'-hydoxymethylation was increased in the context of global hypo-DNAme in vivo, we next analyzed the relationship between the hypo-5me and hyper-5hmC caused by UHRF1 inactivation. In 10 dpp sections, the downregulation of $5 \mathrm{mC}$, but not upregulation of $5 \mathrm{hmC}$, was observed in the mutant spermatocytes. However, both of the two events were clearly observed in the 16 dpp sections (Fig. 7c). The dot blot assay also confirmed that the upregulation of $5 \mathrm{hmC}$ was apparent in the pachytene but not in leptotene stage (Supplementary Fig. S3). In addition, we compared the 3668 upregulated hMeDIP peaks (unique in the mutant spermatocytes) with 11,321 downregulated MeDIP peaks (unique in the control spermatocytes), finding that the overlapping rate was insignificant (Fisher's exact test: $p$ value $=1$ ) (Fig. $7 \mathrm{~d}$ ). Together, our results revealed a spatiotemporal discrepancy of the reduced $5 \mathrm{mC}$ and raised $5 \mathrm{hmC}$ in the spermatocytes with depleted UHRF1. Raised $5 \mathrm{hmC}$ peaks were converted from DNAme sites but not from the hypo-DNAme sites in the mutant spermatocytes.

TET $1-3$ are physiologically downregulated in male meiotic prophase $\mathrm{e}^{22}$. However, we found that TET1, but not TET2 or TET3, was increased gradually in the mutant testis, whereas it was constantly weak from $10 \mathrm{dpp}$ to 16 dpp in the control spermatocytes (Fig. 7e and Supplementary Fig. S4). Note that, in 12 and $16 \mathrm{dpp}$, the meiotic cells with high TET1 level displayed a characteristic of early meiotic cells (leptotene-like, arrowed in green), whereas the cells with low TET1 level displayed a characteristic of later meiotic cells (zygotene or early pachytene-like, arrowed in blue). The upregulation of TET1 provided a rational explanation for this presence of hyper$5 \mathrm{hmC}$ in the spermatocytes without UHRF1. 


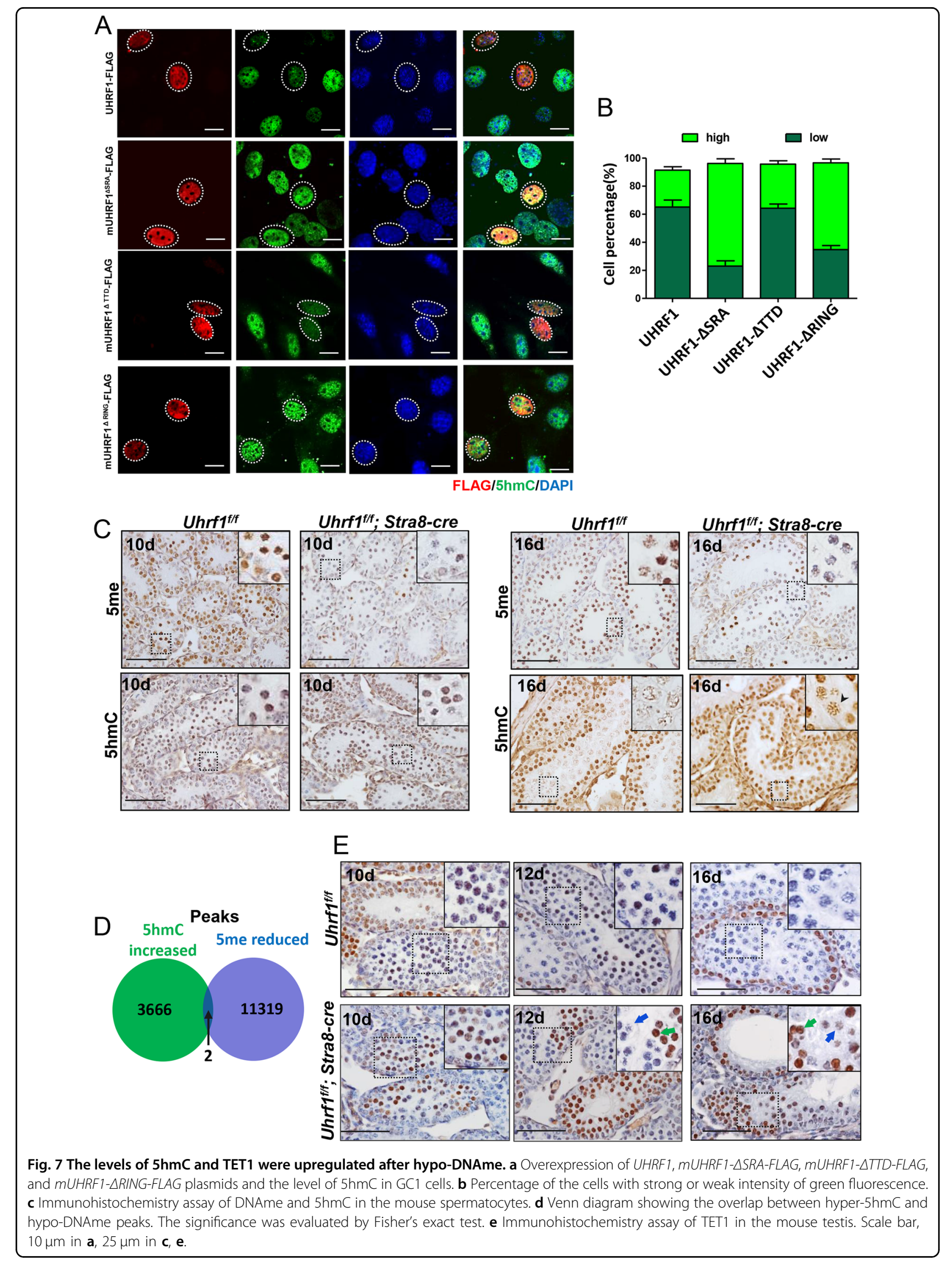




\section{Discussion}

Collectively, we reported that UHRF1 regulated hydroxymethylation in male meiosis. Loss of UHRF1 caused global upregulation of $5 \mathrm{hmC}$. Hyper-5hmC in the TSS region was highly associated with gene repressing in the prophase of meiosis I. In this present study, the repression role of UHRF1 to $5 \mathrm{hmC}$ might partially be the mechanism of physiological downregulation of $5 \mathrm{hmC}$ in male meiotic prophase I.

In our model, an attenuated DNA methylation was detected. We assumed that this is because of the DNA methylation maintenance role of UHRF1and Stra8 expression prior to meiotic prophase. A primary role of DNA methylation is to safeguard the genome via transcriptional silencing of transposable elements during meiosis $^{6,70,71}$. The redundant DMC1 foci and abnormal distribution of $\gamma \mathrm{H} 2 \mathrm{AX}$ in the Uhrf1 knockout spermatocytes strongly implied an impaired genome. A strong activation of RNA retrotransposable elements (for example, SINEs, LINEs, and LTRs) was detected (Supplementary Fig. S5) here. However, we found downregulation of DNA methylation here had little effect on gene transcription increase. A similar conclusion is also reported elsewhere ${ }^{34,72}$. So we think the mechanism of UHRF1 regulating gene transcription in meiosis is different from that in spermatogonium differentiation.

A distinct characteristic of meiosis is a highly protracted cell cycle due to long meiotic $S$ phase and prophase $\mathrm{I}^{73-75}$. During the meiotic $S$ phase and prophase I, a series of profound epigenetic changes occur. The genome-wide $5 \mathrm{mC}$ marks are produced prior to meiotic prophase ( $\mathrm{S}$ phase). Once established, the DNA methylation keeps at relatively high and constant levels in the subsequent stages of meiotic prophase in male germ cells ${ }^{59,72} .5 \mathrm{hmC}$, however, decreases dramatically from preleptotene to pachytene stage. As the influence of $5 \mathrm{hmC}$ distribution on gene expression, it might be possible that such highly ordered $5 \mathrm{hmC}$ dynamic change is required for the spatiotemporal expression of genes in meiosis. By the conditional Uhrf1 knockout mouse model whose spermatocyte DNA $5 \mathrm{hmC}$ level was upregulated, we found that elevated $5 \mathrm{hmC}$ in TSS region might probably contribute to the prevention of RNA-Pol2 binding to downregulate gene expression. However, it should be pointed out that the mechanism of UHRF1 regulating gene expression was complicated. For example, although a peak of $5 \mathrm{hmC}$ was found upregulated at the TSS region (data not shown), the Syce3 gene was increased in the leptotene/zygotene stage. We assumed that this increase would be possibly due to the enhanced levels of transcription factors, as we found that Myc, Fos, and Phf5a were enhanced significantly, and such cis elements were found in the promoter of Syce3 gene in silico (Supplementary Fig. S6).
A number of recent studies in PGCs shows that the lack of DNA methylation maintenance is the main cause of global demethylation ${ }^{76-78}$. A recent study in cultured embryonic stem cells reveals that the impairment of DNA methylation maintenance causes a transient upregulation of $5 \mathrm{hmC}$ in a TET1/2-dependent way ${ }^{79}$. Our model showing the downregulation of DNAme was followed by a global upregulation of $5 \mathrm{hmC}$ in vivo, further implying a synergistic epigenetic regulation of DNA methylation maintenance and hydroxymethylation. Although the specific mechanism of how loss of UHRF1 upregulating $5 \mathrm{hmC}$ is still unclear, we show that TET1 but not TET 2 or TET3 is increased aberrantly. TET1 controls meiosis while its deficiency results in univalent chromosomes and unresolved DSBs in female ${ }^{80}$. In this study, we showed that an aberrant upregulation of TET1 also affected the meiotic progression.

In summary, by the genetic modified mouse model, we pinpointed the dominant role of UHRF1 regulating male meiosis. This study showing UHRF1-repressed $5 \mathrm{hmC}$ highlighted a novel mechanism modifying the epigenetic landscape in spermatogenesis. Although it is unclear currently how UHRF1 targets TET1, we show such repressing role depends on its SRA and RING domains. $5 \mathrm{hmC}$ is one of the reported factors that interact with the SRA domain ${ }^{35}$. Therefore, it is worthy to further study whether $5 \mathrm{hmC}$ is required for the suppressive effect of UHRF1 on TET1 and how SRA and RING domain work synergistically in male meiosis.

\section{Acknowledgements}

This work was supported by grants from the National Natural Science Foundation of China (grant no. 81571495 (to R.L.), 81971443 (to R.L.), 31701303 (to H.P.), 31630050 (to Q.S.), 31890780 (to Q.S.), 31871514 (to X.J.)), InnovationOriented Science and Technology Grant from NHC Key laboratory of Reproduction Regulation (CX2017-07), an Open Project Fund from Key laboratory of Reproduction Regulation of NHC (KF2018-02), and the National Key R\&D Program of China (2018YFC1003502). We also thank Professor Thomas Behnisch and Ms. Yinghan Zhuang for their comments and advice.

\begin{abstract}
Author details
${ }^{1}$ National Health Commission (NHC) Key Laboratory of Reproduction Regulation (Shanghai Institute of Planned Parenthood Research), Fudan University, 200032 Shanghai, P.R. China. ${ }^{2}$ State Key Laboratory of Genetic Engineering, Institute of Biostatistics and Computational Biology, School of Life Sciences, Fudan University, 200438 Shanghai, P.R. China. ${ }^{3}$ The First Affiliated Hospital of USTC, Hefei National Laboratory for Physical Sciences at the Microscale, The CAS Key Laboratory of Innate Immunity and Chronic Diseases, School of Life Sciences, University of Science and Technology of China, 230027 Hefei, Anhui, P.R. China. ${ }^{4}$ East China Normal University and Shanghai Fengxian District Central Hospital Joint Center for Translational Medicine, Shanghai Key Laboratory of Regulatory Biology, School of Life Sciences, East China Normal University, 200241 Shanghai, P.R. China. ${ }^{5}$ Shanghai Endangered Species Conservation and Research Centre, Shanghai Zoo, 200335 Shanghai, P.R. China
\end{abstract}

Conflict of interest

The authors declare that they have no conflict of interest. 


\section{Publisher's note}

Springer Nature remains neutral with regard to jurisdictional claims in published maps and institutional affiliations.

Supplementary Information accompanies this paper at (https://doi.org/ 10.1038/s41419-020-2333-3).

Received: 17 July 2019 Revised: 29 January 2020 Accepted: 30 January 2020 Published online: 21 February 2020

\section{References}

1. Guichaoua, M. R. et al. Meiotic anomalies in infertile men with severe spermatogenic defects. Hum. Reprod. 20, 1897-1902 (2005).

2. Hann, M. C., Lau, P. E. \& Tempest, H. G. Meiotic recombination and male infertility: from basic science to clinical reality? Asian J. Androl. 13, 212-218 (2011).

3. Kota, S. K. \& Feil, R. Epigenetic transitions in germ cell development and meiosis. Dev. Cell 19, 675-686 (2010).

4. Kaneda, M. et al. Essential role for de novo DNA methyltransferase Dnmt3a in paternal and maternal imprinting. Nature 429, 900-903 (2004).

5. Zamudio, N. et al. DNA methylation restrains transposons from adopting a chromatin signature permissive for meiotic recombination. Genes Dev. 29 1256-1270 (2015)

6. Sasaki, H. \& Matsui, Y. Epigenetic events in mammalian germ-cell development: reprogramming and beyond. Nat. Rev. Genet. 9, 129-140 (2008).

7. Jena, S. C. et al. Differential methylation status of IGF2-H19 locus does not affect the fertility of crossbred bulls but some of the CTCF binding sites could be potentially important. Mol. Reprod. Dev. 81, 350-362 (2014).

8. Kuramochi-Miyagawa, S. et al. DNA methylation in mouse testes. Methods Mol. Biol. 1093, 97-109 (2014).

9. Schutte, B. et al. Broad DNA methylation changes of spermatogenesis, inflammation and immune response-related genes in a subgroup of sperm samples for assisted reproduction. Andrology 1, 822-829 (2013).

10. Klaver, R. et al. DNA methylation in spermatozoa as a prospective marker in andrology. Andrology 1, 731-740 (2013).

11. Globisch, D. et al. Tissue distribution of 5-hydroxymethylcytosine and search for active demethylation intermediates. PLOS ONE 5, e15367 (2010).

12. Munzel, M. et al. Quantification of the sixth DNA base hydroxymethylcytosine in the brain. Angew. Chem. 49, 5375-5377 (2010).

13. Chen, Z. X. \& Riggs, A. D. DNA methylation and demethylation in mammals. J. Biol. Chem. 286, 18347-18353 (2011).

14. Jin, S. G. et al. 5-Hydroxymethylcytosine is strongly depleted in human cancers but its levels do not correlate with IDH1 mutations. Cancer Res. 71, 7360-7365 (2011).

15. Song, C. X. et al. Selective chemical labeling reveals the genome-wide distribution of 5-hydroxymethylcytosine. Nat. Biotechnol. 29, 68-72 (2011).

16. Szulwach, K. E. et al. 5-hmC-mediated epigenetic dynamics during postnatal neurodevelopment and aging. Nat. Neurosci. 14, 1607-1616 (2011).

17. Almeida, R. D. et al. Semi-quantitative immunohistochemical detection of 5-hydroxymethyl-cytosine reveals conservation of its tissue distribution between amphibians and mammals. Epigenetics 7, 137-140 (2012).

18. Han, D. et al. A highly sensitive and robust method for genome-wide $5 \mathrm{hmC}$ profiling of rare cell populations. Mol. Cell 63, 711-719 (2016).

19. Diotel, N. et al. 5-hydroxymethylcytosine marks postmitotic neural cells in the adult and developing vertebrate central nervous system. J. Comp. Neurol. 525, 478-497 (2017)

20. Lesch, B. J. \& Page, D. C. Genetics of germ cell development. Nat. Rev. Genet. 13, 781-794 (2012).

21. Hill, P. W. S. et al. Epigenetic reprogramming enables the transition from primordial germ cell to gonocyte. Nature 555, 392-396 (2018).

22. Gan, H. et al. Dynamics of 5-hydroxymethylcytosine during mouse spermatogenesis. Nat. Commun. 4, 1995 (2013).

23. Shirakawa, T. et al. An epigenetic switch is crucial for spermatogonia to exit the undifferentiated state toward a Kit-positive identity. Development $\mathbf{1 4 0}$, 3565-3576 (2013).

24. Kim, K. Y. et al. Uhrf1 regulates active transcriptional marks at bivalent domains in pluripotent stem cells through Setd1a. Nat. Commun. 9, 2583 (2018).
25. DaRosa, P. A. et al. A bifunctional role for the UHRF1 UBL domain in the control of hemi-methylated DNA-dependent histone ubiquitylation. Mol. Cell 72, 753-765. e756 (2018).

26. Foster, B. M. et al. Critical role of the UBL domain in stimulating the E3 ubiquitin ligase activity of UHRF1 toward chromatin. Mol. Cell 72, 739-752. e739 (2018)

27. Liu, X. et al. UHRF1 targets DNMT1 for DNA methylation through cooperative binding of hemi-methylated DNA and methylated H3K9. Nat. Commun. 4, 1563 (2013)

28. Oh, Y. M. et al. Epigenetic regulator UHRF1 inactivates REST and growth suppressor gene expression via DNA methylation to promote axon regeneration. Proc. Natl Acad. Sci. USA 115, E12417-E12426 (2018).

29. Li, Y. et al. Stella safeguards the oocyte methylome by preventing de novo methylation mediated by DNMT1. Nature 564, 136-140 (2018).

30. Yamashita, M. et al. Uhrf1 is indispensable for normal limb growth by regulating chondrocyte differentiation through specific gene expression. Development 145, dev157412 (2018).

31. Choi, M. et al. Epigenetic memory via concordant DNA methylation is inversely correlated to developmental potential of mammalian cells. PLoS Genet. 13, e1007060 (2017).

32. Maenohara, S. et al. Role of UHRF1 in de novo DNA methylation in oocytes and maintenance methylation in preimplantation embryos. PLoS Genet. 13 e1007042 (2017).

33. Zhao, J, et al. Uhrf1 controls the self-renewal versus differentiation of hematopoietic stem cells by epigenetically regulating the cell-division modes. Proc. Natl Acad. Sci. USA 114, E142-E151 (2017).

34. Ramesh, V. et al. Loss of Uhrf1 in neural stem cells leads to activation of retroviral elements and delayed neurodegeneration. Genes Dev. 30, 2199-2212 (2016).

35. Frauer, C. et al. Recognition of 5-hydroxymethylcytosine by the Uhrf1 SRA domain. PLoS ONE 6, e21306 (2011).

36. Lin, Z. et al. Mettl3-/Mettl14-mediated mRNA N(6)-methyladenosine modulates murine spermatogenesis. Cell Res. 27, 1216-1230 (2017).

37. Uphoff, C. C. \& Drexler, H. G. Detecting mycoplasma contamination in cell cultures by polymerase chain reaction. Methods Mol. Biol. 731, 93-103 (2011).

38. Cai, X., Li, J., Yang, Q. \& Shi, Q. Gamma-irradiation increased meiotic crossovers in mouse spermatocytes. Mutagenesis 26, 721-727 (2011).

39. Yang, Q. et al. Synapsis and meiotic recombination in male Chinese muntjac (Muntiacus reevesi). PLoS ONE 6, e19255 (2011).

40. Pimenta, M. T., Francisco, R. A., Silva, R. P., Porto, C. S. \& Lazari, M. F. Relaxin affects cell organization and early and late stages of spermatogenesis in a coculture of rat testicular cells. Andrology 3, 772-786 (2015).

41. Rivarola, M. A., Sanchez, P. \& Saez, J. M. Inhibition of RNA and DNA synthesis in Sertoli cells by co-culture with spermatogenic cells. Int. J. Androl. 9, 424-434 (1986).

42. Mohn, F., Weber, M., Schubeler, D. \& Roloff, T. C. Methylated DNA immunoprecipitation (MeDIP). Methods Mol. Biol. 507, 55-64 (2009).

43. Maunakea, A. K. et al. Conserved role of intragenic DNA methylation in regulating alternative promoters. Nature 466, 253-257 (2010).

44. Baudat, F., Imai, Y. \& de Massy, B. Meiotic recombination in mammals: localization and regulation. Nat. Rev. Genet. 14, 794-806 (2013).

45. Duroc, Y. et al. Concerted action of the MutLbeta heterodimer and Mer3 helicase regulates the global extent of meiotic gene conversion. elife $\mathbf{6}$ e21900 (2017).

46. Martin, A. C., Shaw, P., Phillips, D., Reader, S. \& Moore, G. Licensing MLH1 sites for crossover during meiosis. Nat. Commun. 5, 4580 (2014).

47. Marcet-Ortega, M. et al. p53 and TAp63 participate in the recombinationdependent pachytene arrest in mouse spermatocytes. PLoS Genet. 13 e1006845 (2017).

48. Tachibana, M., Nozaki, M., Takeda, N. \& Shinkai, Y. Functional dynamics of H3K9 methylation during meiotic prophase progression. EMBO J. 26, 3346-3359 (2007).

49. Becherel, O. J. et al. Senataxin plays an essential role with DNA damage response proteins in meiotic recombination and gene silencing. PLoS Genet. $\mathbf{9}$ e1003435 (2013).

50. Pan, $H$. et al. Ndrg3 gene regulates DSB repair during meiosis through modulation the ERK signal pathway in the male germ cells. Sci. Rep. 7, 44440 (2017).

51. Verver, D. E., Langedijk, N. S., Jordan, P. W., Repping, S. \& Hamer, G. The SMC5/6 complex is involved in crucial processes during human spermatogenesis. Biol. Reprod. 91, 22 (2014). 
52. Hwang, G., Verver, D. E., Handel, M. A., Hamer, G. \& Jordan, P. W. Depletion of SMC5/6 sensitizes male germ cells to DNA damage. Mol. Biol. Cell 29, 3003-3016 (2018).

53. Horvath, G. C., Kistler, W. S. \& Kistler, M. K. RFX2 is a potential transcriptional regulatory factor for histone $\mathrm{H} 1 \mathrm{t}$ and other genes expressed during the meiotic phase of spermatogenesis. Biol. Reprod. 71, 1551-1559 (2004).

54. Dunne, O. M. \& Davies, O. R. A molecular model for self-assembly of the synaptonemal complex protein SYCE3. J. Biol. Chem. 294, 9260-9275 (2019).

55. Schramm, S. et al. A novel mouse synaptonemal complex protein is essential for loading of central element proteins, recombination, and fertility. PLoS Genet. 7, e1002088 (2011).

56. Liu, S. et al. Setdb1 is required for germline development and silencing of H3K9me3-marked endogenous retroviruses in primordial germ cells. Genes Dev. 28, 2041-2055 (2014).

57. Manakov, S. A. et al. MIVI2 and MILI have differential effects on piRNA biogenesis and DNA methylation. Cell Rep. 12, 1234-1243 (2015).

58. Jones, P. A. Functions of DNA methylation: islands, start sites, gene bodies and beyond. Nat. Rev. Genet. 13, 484-492 (2012).

59. Gaysinskaya, V. et al. Transient reduction of DNA methylation at the onset of meiosis in male mice. Epigenetics Chromatin 11, 15 (2018).

60. $\mathrm{Wu}, \mathrm{H}$. et al. Genome-wide analysis of 5-hydroxymethylcytosine distribution reveals its dual function in transcriptional regulation in mouse embryonic stem cells. Genes Dev. 25, 679-684 (2011).

61. Robertson, J., Robertson, A. B. \& Klungland, A. The presence of 5hydroxymethylcytosine at the gene promoter and not in the gene body negatively regulates gene expression. Biochem. Biophys. Res. Commun. 411 40-43 (2011).

62. Hsu, L. C. et al. DAZAP1, an hnRNP protein, is required for normal growth and spermatogenesis in mice. RNA 14, 1814-1822 (2008).

63. Zhou, Z. et al. Pold3 is required for genomic stability and telomere integrity in embryonic stem cells and meiosis. Nucleic Acids Res. 46, 3468-3486 (2018).

64. Madura, K. \& Prakash, S. Transcript levels of the Saccharomyes cerevisiae DNA repair gene RAD23 increase in response to UV light and in meiosis but remain constant in the mitotic cell cycle. Nucleic Acids Res. 18, 4737-4742 (1990).

65. Huang, N., Tan, L., Xue, Z., Cang, J. \& Wang, H. Reduction of DNA hydroxymethylation in the mouse kidney insulted by ischemia reperfusion. Biochem. Biophys. Res. Commun. 422, 697-702 (2012).
66. Davis, T. \& Vaisvila, R. High sensitivity 5-hydroxymethylcytosine detection in Balb/C brain tissue. J. Vis. Exp. 48, e2661 (2011).

67. Zheng, $\mathrm{H}$. et al. Genome-wide alteration in DNA hydroxymethylation in the sperm from bisphenol A-exposed men. PLOS ONE 12, e0178535 (2017).

68. Mayer, A. et al. Uniform transitions of the general RNA polymerase II transcription complex. Nat. Struct. Mol. Biol. 17, 1272-1278 (2010).

69. Sidhu, H. \& Capalash, N. UHRF1: The key regulator of epigenetics and molecular target for cancer therapeutics. Tumour Biol. 39, 1010428317692205 (2017).

70. Bourc'his, D. \& Bestor, T. H. Meiotic catastrophe and retrotransposon reactivation in male germ cells lacking Dnmt3L. Nature 431, 96-99 (2004).

71. Ollinger, R., Reichmann, J. \& Adams, I. R. Meiosis and retrotransposon silencing during germ cell development in mice. Differentiation 79, 147-158 (2010).

72. Hammoud, S. S. et al. Chromatin and transcription transitions of mammalian adult germline stem cells and spermatogenesis. Cell Stem Cell 15, 239-253 (2014).

73. Jaramillo-Lambert, A., Ellefson, M., Villeneuve, A. M. \& Engebrecht, J. Differential timing of $S$ phases, $X$ chromosome replication, and meiotic prophase in the $C$. elegans germ line. Developmental Biol. 308, 206-221 (2007).

74. Callan, H. G. \& Taylor, J. H. A radioautographic study of the time course of male meiosis in the newt Triturus vulgaris. J. Cell Sci. 3, 615-626 (1968).

75. Adler, I. D. Comparison of the duration of spermatogenesis between male rodents and humans. Mutat. Res. 352, 169-172 (1996).

76. Seisenberger, $\mathrm{S}$. et al. The dynamics of genome-wide DNA methylation reprogramming in mouse primordial germ cells. Mol. Cell 48, 849-862 (2012).

77. Smith, Z. D. \& Meissner, A. The simplest explanation: passive DNA demethylation in PGCs. EMBO J. 32, 318-321 (2013).

78. Kagiwada, S., Kurimoto, K., Hirota, T., Yamaji, M. \& Saitou, M. Replicationcoupled passive DNA demethylation for the erasure of genome imprints in mice. EMBO J. 32, 340-353 (2013).

79. von Meyenn, F. et al. Impairment of DNA methylation maintenance is the main cause of global demethylation in naive embryonic stem cells. Mol. Cell 62, 983 (2016).

80. Yamaguchi, S. et al. Tet1 controls meiosis by regulating meiotic gene expression. Nature 492, 443-447 (2012). 